\title{
Optical constants of Pluto aerosol analogues from UV to near-IR
}

2 Lora Jovanović ${ }^{1}$, Thomas Gautier ${ }^{1}$, Laurent Broch $^{2}$, Silvia Protopapa ${ }^{3}$, Tanguy Bertrand ${ }^{4}$, 3 Pascal Rannou ${ }^{5}$, Marie Fayolle ${ }^{6,7}$, Eric Quirico ${ }^{6}$, Luc Johann ${ }^{2}$, Aotmane En Naciri ${ }^{2}$, Nathalie 4 Carrasco $^{1}$

5

61 Université Paris-Saclay, UVSQ, CNRS, Sorbonne Université, LATMOS/IPSL, 78280

7 Guyancourt, France

$8 \quad{ }^{2}$ Université de Lorraine, LCP-A2MC, F-57000 Metz, France

$9 \quad{ }^{3}$ Southwest Research Institute, Boulder, CO 80302, USA

${ }^{4}$ NASA Ames Research Center, Moffet Field, CA 94035, USA

${ }^{5}$ GSMA, UMR 7331-GSMA, Université de Reims Champagne-Ardenne, 51687 Reims, France

${ }^{6}$ Université Grenoble Alpes, CNRS, IPAG, 38000 Grenoble, France

${ }^{7}$ Delft University of Technology, The Netherlands

\section{Keywords}

Pluto; Pluto, Aerosols, Haze; Pluto, Atmosphere; Pluto, Surface; Optical Constants

\section{Abstract}

Photochemical aerosols were detected as high as $350 \mathrm{~km}$ of altitude in Pluto's atmosphere during the New Horizons fly-by. These aerosols are thought to affect Pluto's climate, by acting as cooling agents, and the colours of Pluto's surface, in particular in the dark regions named Cthulhu and Krun and at the North Pole. Pluto atmospheric and surface models have so far used the optical constants of Titan aerosol analogues (tholins), whereas their chemical composition is known to differ from that of Pluto aerosol analogues.

In order to provide a new set of optical constants for Pluto tholins, we synthesized analogues of Pluto's aerosols and determined with spectroscopic ellipsometry their optical constants from 270 to $2100 \mathrm{~nm}$. Three types of samples were produced from $\mathrm{N}_{2}: \mathrm{CH}_{4}: \mathrm{CO}$ gas mixtures differing 
in their $\mathrm{CH}_{4}: \mathrm{N}_{2}$ mixing ratio, representative of different altitudes in Pluto's current atmosphere or different seasons or epochs of Pluto.

Our analysis shows a strong absorption by Pluto tholins in the UV and visible spectral ranges, with $k$ index of a few $10^{-1}$ at $270 \mathrm{~nm}$, in agreement with $\mathrm{N}$ - and O-bearing organic molecules. Pluto tholins are less absorbent in the near-IR than in the UV-Vis wavelength range, with $k$ of a few $10^{-3}$ between 600 and $2100 \mathrm{~nm}$. Our comparative study highlights the dependency of $n$ and $k$ indices to the $\mathrm{CH}_{4}: \mathrm{N}_{2}$ mixing ratio. Aerosols formed at different altitudes in Pluto's atmosphere or during different seasons or epochs of Pluto will therefore affect the budget of Pluto radiative transfer differently.

The optical constants presented in this study were tested with a Pluto surface model and with a model of light scattering. The surface modelling results highlight the suitability of these optical constants to reproduce Pluto compositional observations in the visible spectral range by MVIC and LEISA. The atmospheric modelling results conclude that Pluto tholins absorb 5 to 10 times less than Titan tholins at $500 \mathrm{~nm}$, and this lower absorption is consistent with Alice observations of Pluto's haze.

\section{Introduction}

Pluto fly-by by the New Horizons spacecraft on July 14 ${ }^{\text {th }}$, 2015 evidenced the presence of aerosols in Pluto's atmosphere by means of forward scattering observations and solar occultations. These aerosols, accounting for $0.05-0.1 \mathrm{ppmv}$ of the atmosphere, enshroud Pluto and were detected at least as high as $350 \mathrm{~km}$ of altitude above the surface (Cheng et al. 2017; Gladstone et al. 2016; Stern et al. 2015; Young et al. 2018).

Two mechanisms are currently proposed for the formation of hazes in Pluto's atmosphere.

The first one concerns the formation of aerosols through photochemistry, similar to what occurs in Titan's upper atmosphere (e.g., Gladstone et al. 2015, 2016; Wong et al. 2017; Young et al. 2018). Pluto's aerosols are thought to be formed by a complex photochemistry taking place in the upper atmosphere, below $300 \mathrm{~km}$ and up to $900 \mathrm{~km}$ of altitude (Stern et al. 2018; Young et al. 2018). This photochemistry is initiated by extreme-ultraviolet (EUV) sunlight and solar Lyman- $\alpha$ photons. EUV radiation breaks the triple bonds of $\mathrm{N}_{2}$ and $\mathrm{CO}$ in the upper atmosphere, while Lyman- $\alpha$ photons - arriving directly from the Sun or scattered by the interplanetary medium - breaks the $\mathrm{C}-\mathrm{H}$ bonds in $\mathrm{CH}_{4}$ at lower altitudes (Gladstone et al. 2015, 2016; Grundy 
et al. 2018, and references therein), with a maximum $\mathrm{CH}_{4}$ photolysis rate at $250 \mathrm{~km}$ of altitude above the surface (Bertrand \& Forget 2017). Pluto's photochemistry involves neutral and ionic species created by the ionization and the dissociation of molecular nitrogen $\mathrm{N}_{2}$, methane $\mathrm{CH}_{4}$, and carbon monoxide CO that constitute Pluto's atmosphere (Gladstone et al. 2016; Lellouch et al. 2017; Young et al. 2018). This chemistry forms complex hydrocarbons, nitriles, and oxygenated molecules, including some that can further polymerize and generate solid aerosols (Krasnopolsky 2020; Wong et al. 2017).

The second mechanism, developed by Lavvas et al. (2020), would be different from that proposed for Titan's hazes formation, due to the temperature difference between Pluto's and Titan's upper atmospheres ( $\sim 70 \mathrm{~K}$ versus $\sim 150 \mathrm{~K}$, respectively). This mechanism involves the direct condensation of photochemical gases produced on Pluto, associated with a coating by undersaturated light molecules ( $\mathrm{C}_{2}$ hydrocarbons) incorporated by heterogeneous processes (possibly chemisorption or physisorption). This coating represents $\sim 30 \%$ of the aerosol material (Lavvas et al. 2020). These coated aerosols are then subject to a photon flux causing their ageing, as studied in Carrasco et al. (2018) for Titan's aerosols.

Note that the present study focuses on Pluto's aerosols formed by photochemistry.

Questions remain regarding the size distribution of Pluto's haze particles since no single solution can satisfy measurements from all instruments onboard New Horizons. Cheng et al. (2017) pointed out the contradiction between haze brightness I/F profiles inferred from Alice imaging spectrograph in the far-UV [52-187 nm] (Stern et al. 2008) and LORRI (LOng-Range Reconnaissance Imager) panchromatic imaging instrument in the [350-850 nm] wavelength range (Cheng et al. 2008). A model of Mie scattering by $0.5 \mu \mathrm{m}$ spheres reproduces fairly well the phase function of Pluto's haze at peak I/F measured in the visible by LORRI. However, this Mie model gives UV extinction lower by an order of magnitude than that retrieved from Alice solar occultation. On the opposite, results from a model of scattering by aggregates with bulk radius of $0.15 \mu \mathrm{m}$ composed of several thousands $0.01 \mu \mathrm{m}$ spherical monomers agree with the measurements made by Alice. They also match with the visible phase function measured by LORRI, but only at higher altitudes in Pluto's atmosphere (Cheng et al. 2017). At higher altitudes, Pluto's haze is thus thought to be mostly composed of aggregates of $0.15 \mu \mathrm{m}$ particles made up of $10 \mathrm{~nm}$ forward-scattering spheres. The haze particles present in the atmosphere from the surface to $\sim 15 \mathrm{~km}$ of altitude probably correspond to spherical particles that got larger by direct condensation of photochemical products onto nuclei (Cheng et al. 2017; Gao et al. 2017; Gladstone et al. 2016; Lavvas et al. 2020; Stern et al. 2015; Young et al. 2018). 
The haze particles are suspected to scatter the UV sunlight and to be the dominant factor of extinction at wavelengths greater than $\sim 150 \mathrm{~nm}$, as the extinctions of hydrocarbons or nitriles become less important with increasing wavelength. The haze particles thus contribute to the spectrum of Pluto observed by the Alice instrument (Cheng et al. 2017; Steffl et al. 2020; Stern et al. 2008). Pluto's aerosols are also expected to significantly absorb in the UV-Visible and in the far-infrared spectral ranges and to be less absorbent in the near- and mid-IR (Grundy et al. 2018; Zhang et al. 2017; and references therein).

Recent numerical models have shown that these aerosols deeply affect Pluto's atmospheric chemistry, for instance by depleting from the atmosphere small hydrocarbons that stick to the negatively-charged aerosols, resulting in the formation of more complex ones (Krasnopolsky 2020; Luspay-Kuti et al. 2017; Wong et al. 2017). Pluto's climate is also affected by the aerosols, that can serve as condensation nuclei (Gao et al. 2017; Lavvas et al. 2016; LuspayKuti et al. 2017; Wong et al. 2017) or through direct radiative cooling of the atmosphere by the absorption of solar radiations (Zhang et al. 2017). Pluto's atmosphere temperature, at $\sim 400 \mathrm{~km}$ of altitude, is around $30-40 \mathrm{~K}$ colder than model predictions for an atmosphere in radiativeconductive equilibrium without haze (Gladstone et al. 2016; Zhang et al. 2017; Zhu et al. 2014). Zhang et al. (2017) suggested this cooling to be due to the haze particles and their optical properties. Due to a lack of both observational and experimental data on these optical properties, the study by Zhang et al. (2017) was done using those of Titan aerosol analogues (Titan tholins) produced on Earth. In addition, these photochemical aerosols have been suggested to account for all of Pluto's colours at large scales, from the dark red colour of Cthulhu and Krun Maculae to the yellow hue on Pluto's North Pole (Lowell Regio) (Grundy et al. 2018; Protopapa et al. 2017, 2020). The modelling studies of Pluto New Horizons data across the full [0.4-2.5 $\mu \mathrm{m}]$ wavelength range by Protopapa et al. (2020) used optical constants determined for Titan tholins by Khare et al. (1984) and Tran et al. (2003), given the lack of optical constants for Pluto tholins. To explain the different colours on Pluto's surface, Grundy et al. (2018) used multiplescattering radiative transfer models with optical constants determined for Titan tholins by several groups: Khare et al. (1984), Ramírez et al. (2002), Imanaka et al. (2004), Vuitton et al. (2009), and Sciamma-O’Brien et al. (2012).

Protopapa et al. (2020) pointed out the lack of optical constants for tholins in the Vis-near-IR wavelength range. Additionally, Zhang et al. (2017) highlighted that the optical constants determined for Titan aerosol analogues strongly vary with the mode of production and that the optical constants of Pluto's aerosols may be very different from those of Titan tholins. Note 
that the review by Brassé et al. (2015) discusses the different optical constants determined for Titan tholins.

Thus, to update and better constrain Pluto atmospheric models and to model Pluto's surface spectra, the determination of the optical constants of Pluto aerosol analogues is strongly needed. The aim of our work is to fill this gap, by providing optical constants of Pluto tholins from 270 to $2100 \mathrm{~nm}$.

\section{Experimental setup and protocol}

\section{Production of Pluto aerosol analogues}

Following the protocol detailed in Jovanović et al. (2020), we used the experimental setup PAMPRE (Production d'Aérosols en Microgravité par Plasma REactif) (Alcouffe et al. 2010; Szopa, Cernogora et al. 2006), a cold plasma discharge to simulate the chemistry occurring in Pluto's atmosphere and produce analogues of Pluto's aerosols. The gas mixture flows through a cylindrical metallic grounded cage. Square silicon wafers of $1 \mathrm{~cm}^{2}$ were placed at the bottom of the cage. When the plasma is switched on, tholins form as thin films onto the silicon wafers.

Note that the optical constants of tholins are highly dependent on the experimental conditions of synthesis and measurements (Brassé et al. 2015; Zhang et al. 2017). For readers interested in the effect of using a UV lamp rather than a plasma discharge to synthesize tholins, we recommend reading the review by Cable et al. (2012). In summary, tholins formed by UV photochemistry in the laboratory tend to be less rich in N-bearing compounds, since UV lamps cannot mobilize nitrogen chemistry. Indeed, lamp windows cut off UV wavelengths below $\sim 115 \mathrm{~nm}$, which prevents direct dissociation of $\mathrm{N}_{2}$, unlike what happens in natural atmospheres. On the contrary, plasma setups such as PAMPRE tend to enhance nitrogen inclusion a bit too much. The electron energy distribution in PAMPRE mimics well at first order the one of solar photons (Szopa, Cernogora et al. 2006). However, a small difference is due to the slightly more energetic tail of the Maxwellian energy distribution of the electrons in the plasma compared to the Planck energy distribution of solar photons. This slightly higher population of electrons induces a small bias due to over-dissociation of $\mathrm{N}_{2}$, that is well known and has been extensively studied regarding the tholins chemical composition. Since nitrogenous compounds tend to be more absorbent, one could expect that tholins synthesized in laboratory by UV photochemistry would probably be less absorbent in the UV-Vis spectral range, due to a poorer content in Nbearing compounds. Where actual Pluto's aerosols would stand in that range is currently unknown (as for Titan's aerosols), which is why we strongly encourage any other experimental 
teams with similar facilities to perform studies similar to ours to extend the range of possible laboratory data on this point.

Three types of tholins were synthesized in this work, from gas mixtures composed of various $\mathrm{N}_{2}$ (Air Liquide, ALPHAGAZTM 2) and $\mathrm{CH}_{4}$ (Air Liquide, CRYSTAL mixture) percentages and a fixed CO (Air Liquide, CRYSTAL mixture) amount of $500 \mathrm{ppm}$ (see Table 1), at $0.9 \pm 0.1$ mbar and ambient temperature. For readers interested in the effect of synthesis temperature on aerosol analogues optical constants, we recommend reading Mahjoub et al. (2014) who studied this effect for Titan tholins. In the present work, experiments were performed at ambient temperature to avoid heterogeneous chemistry effects as described in Mahjoub et al. (2014).

The CO atmospheric mole fraction of $515 \pm 40$ ppm (Lellouch et al. 2017; Young et al. 2018) is not expected to vary much over time and space. This is due to the volatility of CO being close to that of $\mathrm{N}_{2}$ and the absence of CO-rich ice deposits on Pluto's surface (Bertrand \& Forget 2016). The first type of tholins was produced in a gas mixture composed of $\mathrm{N}_{2}: \mathrm{CH}_{4}: \mathrm{CO}$ at 99.5\%:0.5\%:500ppm - we will thereafter call this material " $\mathrm{P}_{\mathrm{H}}$ ". Given that the $\mathrm{CH}_{4}$ mixing ratio was found to be $\sim 0.3 \%$ below $350 \mathrm{~km}$ of altitude (Young et al. 2018), $\mathrm{P}_{\mathrm{H}}$ is relevant to the composition of Pluto's atmosphere as observed in 2015 below $350 \mathrm{~km}$ of altitude above the surface. The second tholins sample was produced in a gas mixture containing $\mathrm{N}_{2}: \mathrm{CH}_{4}: \mathrm{CO}$ at 99\%:1\%:500ppm, mimicking Pluto's atmosphere at around $400 \mathrm{~km}$ of altitude above the surface - thereafter called " $\mathrm{P}_{400}$ ". The third one was produced in a gas mixture made of $\mathrm{N}_{2}: \mathrm{CH}_{4}: \mathrm{CO}$ at 95\%:5\%:500ppm, representative of Pluto's atmosphere between 600 and $700 \mathrm{~km}$ of altitude - thereafter called " $\mathrm{P}_{650}$ " (Lellouch et al., 2017, Young et al., 2018).

We note that the mean $\mathrm{CH}_{4}$ atmospheric mixing ratio is predicted to vary from $0.01 \%$ to $5 \%$ over annual or astronomical timescales (Bertrand et al. 2019; Bertrand \& Forget 2016). The tholins synthesized in the present work could therefore also serve as analogues for haze particles produced during past epochs or different seasons of Pluto, with implications to its past and future climates.

For the three types of tholins, the plasma ran for 90 minutes, forming films with thicknesses no more than $1 \mu \mathrm{m}$ (see Table 1) to ensure the validity of the spectroscopic ellipsometry technique for the measurements (Fujiwara 2007; Mahjoub et al. 2012; Sciamma-O'Brien et al. 2012). 
Table 1: Table presenting the three types of Pluto aerosol analogues analysed in this study.

\begin{tabular}{|c|c|c|c|c|c|}
\hline \multicolumn{2}{|c|}{ Composition of the reactive } & $\begin{array}{c}\text { Corresponding } \\
\text { altitude above the } \\
\text { mixture }\end{array}$ & $\begin{array}{c}\text { Acronym of } \\
\text { the aerosol } \\
\text { surface on Pluto }\end{array}$ & $\begin{array}{c}\text { Thickness of the } \\
\text { analogues }\end{array}$ & thin film \\
\hline $\mathrm{N}_{2}$ & $\mathrm{CH}_{4}$ & $\mathrm{CO}$ & $<350 \mathrm{~km}$ & $\mathrm{P}_{\mathrm{H}}$ & $549.8 \pm 0.6 \mathrm{~nm}$ \\
\hline $99.5 \%$ & $0.5 \%$ & $500 \mathrm{ppm}$ & $\sim 400 \mathrm{~km}$ & $\mathrm{P}_{400}$ & $540.9 \pm 0.2 \mathrm{~nm}$ \\
\hline $99 \%$ & $1 \%$ & $500 \mathrm{ppm}$ & $\sim 400-700 \mathrm{~km}$ & $\mathrm{P}_{650}$ & $651.0 \pm 0.7 \mathrm{~nm}$ \\
\hline $95 \%$ & $5 \%$ & $500 \mathrm{ppm}$ & 600 & & \\
\hline
\end{tabular}

The thickness of the thin films was determined by spectroscopic ellipsometry with a modified Tauc-Lorentz dispersion model, as detailed below. We note that for the same synthesis duration, the thin-film thickness increases when the amount of $\mathrm{CH}_{4}$ injected in the reactive gas mixture increases significantly, as discussed by Mahjoub et al. (2012).

\section{Spectroscopic ellipsometry}

The tholins thin films were analysed by spectroscopic ellipsometry. Using this analysis technique, it is possible to determine the thickness of the tholins thin films, as well as their optical constants $n$ and $k$, the real and imaginary parts respectively of the complex refractive index.

Spectroscopic ellipsometry measures the change in the polarization state between incident and reflected light on the sample (the reader is referred to e.g., Azzam \& Bashara (1977), Tompkins \& Irene (2005) and Fujiwara (2007) for complete information on spectroscopic ellipsometry technique). Incident light is linearly polarized, while reflected light is elliptically polarized.

The measured values are the ellipsometric parameters $\Psi$ and $\Delta$, related to the ratio of the Fresnel amplitude reflection coefficients of the sample by fundamental equation:

$$
r_{\mathrm{p}} / \mathrm{r}_{\mathrm{s}}=\tan \psi \mathrm{e}^{i \Delta}
$$

for $\mathrm{p}$ - and s-polarized light ${ }^{1}$ with the electric field in the plane of incidence and perpendicular to the plane of incidence, respectively.

\footnotetext{
${ }^{1} r_{p}$ is the reflection coefficient of the sample for a polarization parallel to the plane of incidence (" $\mathrm{p}$ " for parallel) and $r_{s}$ is the reflection coefficient of the sample for a polarization perpendicular to the plane of incidence ("s" for perpendicular, "senkrecht" in German).
} 
Variable Angle Spectroscopic Ellipsometry (VASE) measurements were carried out using a phase-modulated spectroscopic ellipsometer (UVISEL, Horiba Jobin Yvon), equipped with a $150 \mathrm{~W}$ Xenon light source. Corresponding ellipsometric parameters were obtained through $\left(\mathrm{I}_{\mathrm{s}}(\lambda), \mathrm{I}_{\mathrm{c}}(\lambda)\right)$ in the spectral range [270-2100 nm] with $5 \mathrm{~nm}$ steps according an integration time of $1000 \mathrm{~ms}$, where $\mathrm{I}_{\mathrm{s}}=\sin (2 \Psi) \sin (\Delta)$ and $\mathrm{I}_{\mathrm{c}}=\sin (2 \Psi) \cos (\Delta)$, respectively. All measurements were performed for incidence angles between 60 and 75 degrees with $5^{\circ}$ steps. By way of comparison, the ellipsometric analyses performed on Titan tholins thin films by Mahjoub et al. (2012, 2014) and Sciamma-O'Brien et al. (2012) were conducted for a single incidence angle of $70^{\circ}$.

The parameters of the optical model were adjusted by minimizing the Mean Square Error (MSE) defined as:

$$
\operatorname{MSE}=\frac{1}{\mathrm{~N}-1} \sum_{\mathrm{i}=1}^{\mathrm{N}}\left[\left(\mathrm{I}_{\mathrm{s}, \mathrm{i}}^{\mathrm{calc}}(\lambda)-\mathrm{I}_{\mathrm{s}, \mathrm{i}}^{\mathrm{exp}}(\lambda)\right)^{2}+\left(\mathrm{I}_{\mathrm{c}, \mathrm{i}}^{\mathrm{calc}}(\lambda)-\mathrm{I}_{\mathrm{c}, \mathrm{i}}^{\mathrm{exp}}(\lambda)\right)^{2}\right]
$$

where calc stands for the calculated and $\exp$ for the experimental values of $\left(\mathrm{I}_{\mathrm{s}}, \mathrm{I}_{\mathrm{c}}\right)$ and $N$ the number of experimental data $\left(I_{s}(\lambda), I_{c}(\lambda)\right)$.

DeltaPsi $2^{\circledR}$ software $^{2}$ was used to fit ellipsometric data.

The inversion of ellipsometric data was performed using a model of the sample [Si substrate / Tholins layer / Roughness layer / Air] as schematized in Figure 1.

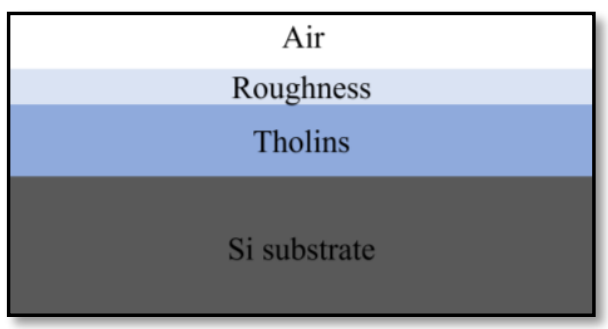

Figure 1: Scheme of the model of the samples analysed by spectroscopic ellipsometry.

In this model, the thickness of the roughness layer, the thickness of the tholins layer, and the dielectric function of the tholins layer were unknown, while the roughness layer was modelled by a mixture of 50\% air and 50\% tholins, according to Bruggeman Effective Medium Approximation (BEMA) (Bruggeman 1935).

\footnotetext{
${ }^{2}$ DeltaPsi2 ${ }^{\circledR}$ software: https://www.horiba.com/en en/products/detail/action/show/Product/deltapsi2-software$\underline{1648 /}$
} 


\section{Determination of the optical constants of Pluto tholins with two different inversion} methods: modified Tauc-Lorentz and "wavelength-by-wavelength" $(\lambda$-by- $\lambda)$

Spectroscopic ellipsometry is an indirect technique for determination of the sample physical properties (i.e. thickness and optical constants). Using a model to describe the ellipsometric response of the sample by adjusting specific fit parameters is thus necessary. Basically, the model calculates the reflection coefficients $r_{p}$ and $r_{s}$ to retrieve the measured ellipsometric parameters $\Psi$ and $\Delta$. An equation system for each wavelength $\lambda$ is generated: $\Psi=\mathrm{f}(\mathrm{n}, \mathrm{k}, \mathrm{t})$ and $\Delta=\mathrm{g}(\mathrm{n}, \mathrm{k}, \mathrm{t})$, with $n(\lambda)$ and $k(\lambda)$ the real and imaginary parts respectively of the complex refractive index, and $t$ the thickness of the tholins thin film. The thickness $t$ of the thin film and the $n$ index are initially determined in a transparent region of the thin film. Then, the fixed $t$ value is used as an input parameter to determine $n$ and $k$ indices in an absorbing region of the thin film. The inversion method, based on the natures of the analysed material and the underlying substrate, is used to calculate $n$ and $k$ indices of the material for each wavelength.

In this work, we used sequentially two types of inversion method: modified Tauc-Lorentz dispersion model to find the proper estimates of the initialization parameters, and "wavelengthby-wavelength" ( $\lambda$-by- $\lambda$ ) numerical inversion to determine precisely the optical constants $n$ and $k$ of the tholins material.

\section{a. Determination of the thickness and the optical constants of Pluto tholins by a} modified Tauc-Lorentz dispersion model

The first model we used is a Tauc-Lorentz dispersion model, based on the Tauc joint density of states and the Lorentz oscillator, which was developed by Jellison and Modine (1996). This dispersion model was developed to better describe the optical properties of semiconductor and amorphous materials. This fitting model is thus adapted for tholins produced with the PAMPRE experimental setup, as Hadamcik et al. (2009) observed these samples with Scanning Electron Microscopy and showed that they present an amorphous structure.

In this model, the imaginary part $\varepsilon_{i, \mathrm{TL}}$ of the complex relative dielectric constant is given by the product of the imaginary part of Tauc's dielectric constant $\varepsilon_{i, T}$ (Tauc et al. 1966) with a Lorentzian oscillator $\varepsilon_{i, \mathrm{~L}}$ :

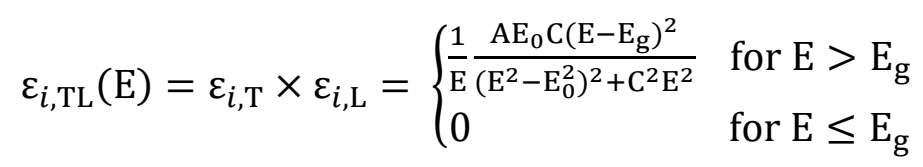


where $\mathrm{E}$ (in $\mathrm{eV}$ ) is the photon energy; $\mathrm{E}_{\mathrm{g}}($ in $\mathrm{eV}$ ) is the optical bandgap energy specifying at which energy the material becomes absorbent; $\mathrm{E}_{0}(\mathrm{in} \mathrm{eV})$ is the energy of the major absorption peak in the UV (the Lorentz peak) $\left(\mathrm{E}_{\mathrm{g}}<\mathrm{E}_{0}\right)$; $\mathrm{A}$ is the oscillator amplitude; and $\mathrm{C}$ is the broadening term.

The real part $\varepsilon_{r, \mathrm{TL}}$ of the complex relative dielectric constant is derived from the expression of $\varepsilon_{i, \mathrm{TL}}$ (Equation (3)) using the Kramers-Kronig integration (Kramers 1927; Kronig 1926):

$$
\varepsilon_{r, \mathrm{TL}}(\mathrm{E})=\varepsilon_{\infty}+\frac{2}{\pi} \cdot \mathrm{P} \cdot \int_{\mathrm{E}_{\mathrm{g}}}^{\infty} \frac{\xi \cdot \varepsilon_{i \mathrm{TL}}(\xi)}{\xi^{2}-\mathrm{E}^{2}} \mathrm{~d} \xi
$$

where $\varepsilon_{\infty}$ is the high-frequency real dielectric constant; and $\mathrm{P}$ is the Cauchy principal value.

The optical constants $n$ and $k$ are then deduced from the real and imaginary parts of the complex relative dielectric constant (Equations (3) and (4)) by the relation:

$$
\varepsilon_{r, \mathrm{TL}}+i \times \varepsilon_{i, \mathrm{TL}}=(n+i \times k)^{2}
$$

In the standard Tauc-Lorentz model, the imaginary part $k$ of the complex refractive index becomes null for energies below the bandgap energy. This is due to the resolution of KramersKronig equations, but not to the intrinsic material properties. However, this $k$ index is necessary for numerical models (e.g., radiative transfer, albedo, surface composition, haze vertical profile).

To overcome this problem, we modified the standard Tauc-Lorentz model by adding oscillators characterized by $\varepsilon_{\text {osc: }}$ :

$$
\varepsilon_{\mathrm{osc}}=\frac{\mathrm{f}_{\mathrm{j}} w_{0 \mathrm{j}}^{2}}{w_{0 \mathrm{j}}^{2}-w^{2}+i \gamma_{\mathrm{j}} w}
$$

where $\varepsilon_{\mathrm{osc}}$ is the dielectric function; $w$ is the light pulsation; $\mathrm{f}_{\mathrm{j}}$ is the oscillator strength of the $\mathrm{j}^{\text {th }}$ resonator; $w_{0 \mathrm{j}}$ is the natural pulsation of the $\mathrm{j}^{\text {th }}$ resonator; and $\gamma_{\mathrm{j}}$ is the damping rate of the $\mathrm{j}^{\text {th }}$ resonator (friction coefficient).

Oscillators were added one by one to the model at different wavelengths, until the addition of an additional oscillator had no longer significant improvement in the MSE (Equation (2)). In this work, although the modified Tauc-Lorentz model was adapted for the determination of the thin-films thickness, the addition of oscillators was not sufficient to retrieve $k$ indices below the bandgap energy (see Figure 3). 

inversion method

Once the thickness of the thin films and the initial couples $\left[n_{270 n m}, k_{270 n m}\right]$ characterized by the modified Tauc-Lorentz dispersion model (values used as input parameters), we used the " $\lambda$-by$\lambda$ " numerical inversion method to determine the optical constants of Pluto tholins.

In the " $\lambda$-by- $\lambda$ " inversion method (also known as "direct data inversion" or "exact numerical inversion"), $n$ and $k$ indices are calculated for each wavelength by applying mathematical inversion of the Equation (1) (Fujiwara 2007). Theoretical ellipsometric parameters are calculated ( $\Psi_{\text {calc }}$ and $\left.\Delta_{\text {calc }}\right)$ and the values are compared to the experimentally measured ellipsometric parameters ( $\Psi_{\exp }$ and $\left.\Delta_{\exp }\right)$. A linear regression algorithm introduces for the initial couple $\left[n_{270 n m}, k_{270 n m}\right]$ the corrections needed to match ( $\left.\Psi_{\text {calc }}, \Delta_{\text {calc }}\right)$ and ( $\left.\Psi_{\exp }, \Delta_{\exp }\right)$. Then, the process is applied iteratively over the whole spectral range (from 270 to $2100 \mathrm{~nm}$ ): the values for the couple $\left[n_{\lambda}, k_{\lambda}\right]$ are calculated from the values of the couple $\left[n_{\lambda-\delta \lambda}, k_{\lambda-\delta \lambda}\right]$ with $\delta \lambda$ the wavelength step between two measurements (here, $5 \mathrm{~nm}$ ) (Fujiwara 2007; Keita et al. 2010; Likhachev et al. 2015).

The " $\lambda$-by- $\lambda$ " numerical inversion method gives a mathematical solution for $\left[n \lambda, k_{\lambda}\right]$ couples from measured ellipsometric parameters $\Psi$ and $\Delta$, disregarding the $n$ and $k$ dependency on each other at different wavelengths. For this procedure, other parameters, such as the thickness of the thin film, and the optical properties of the underlying silicon substrate and the overlying roughness layer, need to be accurately known, otherwise it can lead to multiple solutions, unphysical dispersion or discontinuities in the optical constants, generally present at lower energies in the inversion spectrum (Fujiwara 2007; Keita et al. 2010; Likhachev et al. 2015).

\section{c. Procedure to determine the optical constants $n$ and $k$ of Pluto tholins}

In summary, modified Tauc-Lorentz dispersion model is used to extract both thickness and optical constants $n$ and $k$ by fitting the thickness values and the Tauc-Lorentz parameters. " $\lambda$ by- $\lambda$ " numerical inversion enables an exact modelling of the optical constants $n$ and $k$ in spectral ranges where the fit is difficult with dispersion formulas and to check the optical constants results obtained by dispersion formulas (modified Tauc-Lorentz in this work). A " $\lambda$-by- $\lambda$ ” fit of the experimental data is performed on the only unknown optical constants $n$ and $k$ of the thin film without fitting any parameter. To use this numerical inversion method, all parameters of the optical model, such as thicknesses, must be known, which is not the case for tholins material and thus induces much larger uncertainties. This method requires a (even rough) knowledge of 
the order of magnitude of $n$ and $k$ indices for initialization. We thus used a two-pronged approach, where the modified Tauc-Lorentz dispersion model is used to determine the initialization parameters of the " $\lambda$-by- $\lambda$ " numerical inversion method, which in return gives us the optical properties with much higher confidence. Therefore, our procedure, based on two sequential modified Tauc-Lorentz and " $\lambda$-by- $\lambda$ " methods, is a good way to determine correctly the optical constants $n$ and $k$ of the original sample system when the optical responses are not available in the literature. The reader is referred to Keita et al. (2010) for more details about these two methods.

\section{Results: Optical constants of Pluto aerosol analogues}

Figures 2 and 3 respectively display the real and imaginary parts $n$ and $k$ of the complex refractive index determined for $\mathrm{P}_{\mathrm{H}}$ sample with a modified Tauc-Lorentz dispersion model (in turquoise blue) and with a " $\lambda$-by- $\lambda$ " numerical inversion method (in dark blue). The $2 \sigma$ error bars determined for the " $\lambda$-by- $\lambda$ " inversion method (see Table SI.4 in Supplementary Information) were calculated based on the uncertainties of $\mathrm{I}_{s}^{\text {exp }}$ and $\mathrm{I}_{c}^{\text {exp }}$ at different wavelengths. The uncertainties were then linearly interpolated between two error bars in order to obtain an envelope of uncertainty (in light blue). Note that these error bars do not include all the uncertainties on $n$ - and $k$-values. Indeed, the uncertainties on the raw ellipsometric measurements before modelling are known and are modest, given the quality of the setting of the analytical instrument; these uncertainties are therefore not included in the error bars presented. The major source of uncertainties corresponds to the inversion model used, including e.g., the number of fitting parameters and of oscillators used, the problem of presence of local minima during the fit, and in particular the absence of initial values for the inversion. We can nevertheless expect that the actual uncertainties on the optical constants of Pluto tholins would be slightly higher than those presented here. 


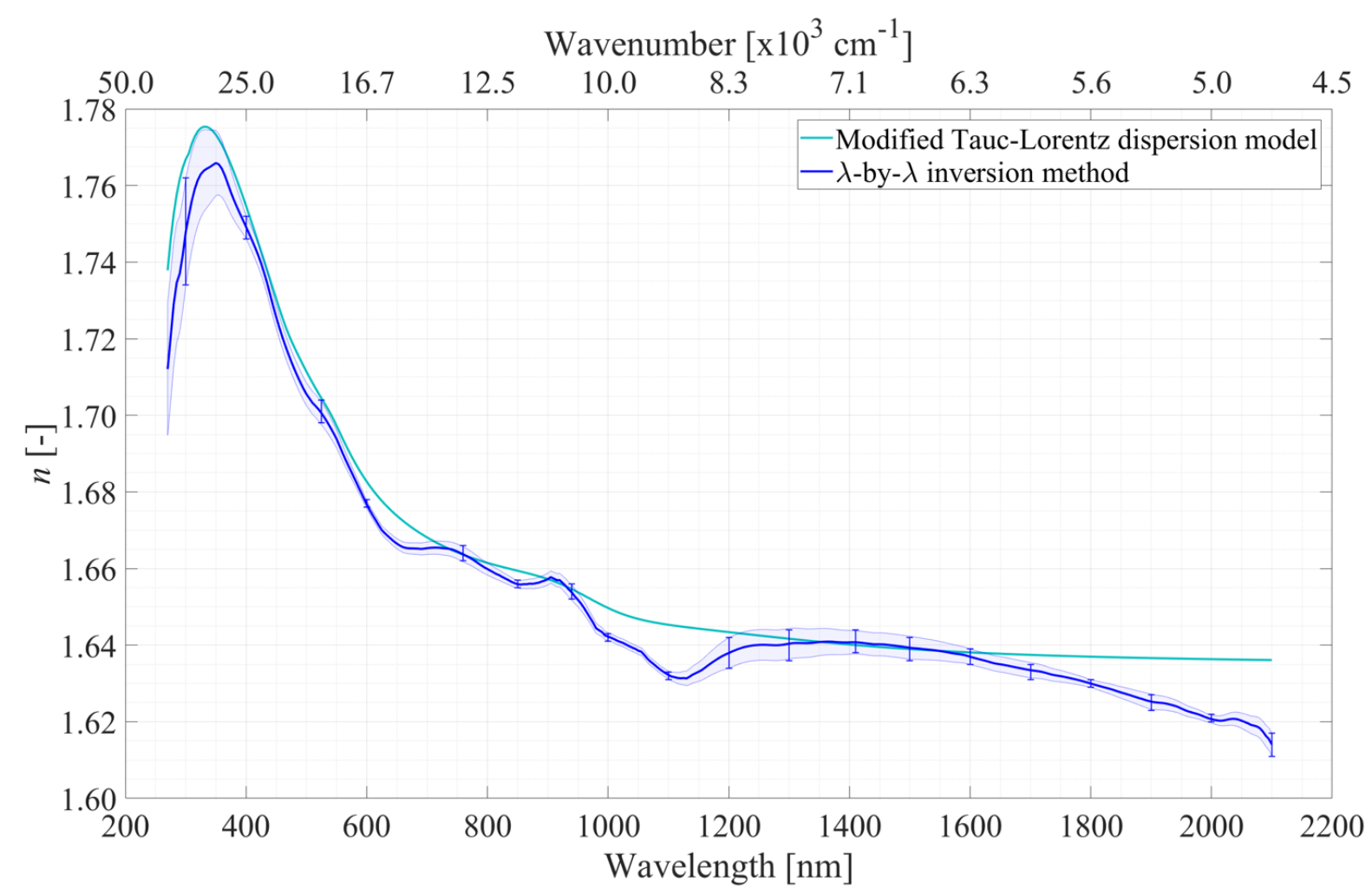

351 Figure 2: Real part $n$ of the complex refractive index of Pluto aerosol analogues $\mathrm{P}_{\mathrm{H}}$ determined by 352 spectroscopic ellipsometry with a modified Tauc-Lorentz dispersion model (in turquoise blue) and with 353 a " $\lambda$-by- $\lambda$ " numerical inversion method (in dark blue). The $2 \sigma$ error bars were experimentally determined 354 for the " $\lambda$-by- $\lambda$ " inversion method at different wavelengths. The uncertainties were then linearly 355 interpolated between two error bars in order to obtain the envelope of $2 \sigma$ uncertainty (in light blue). 


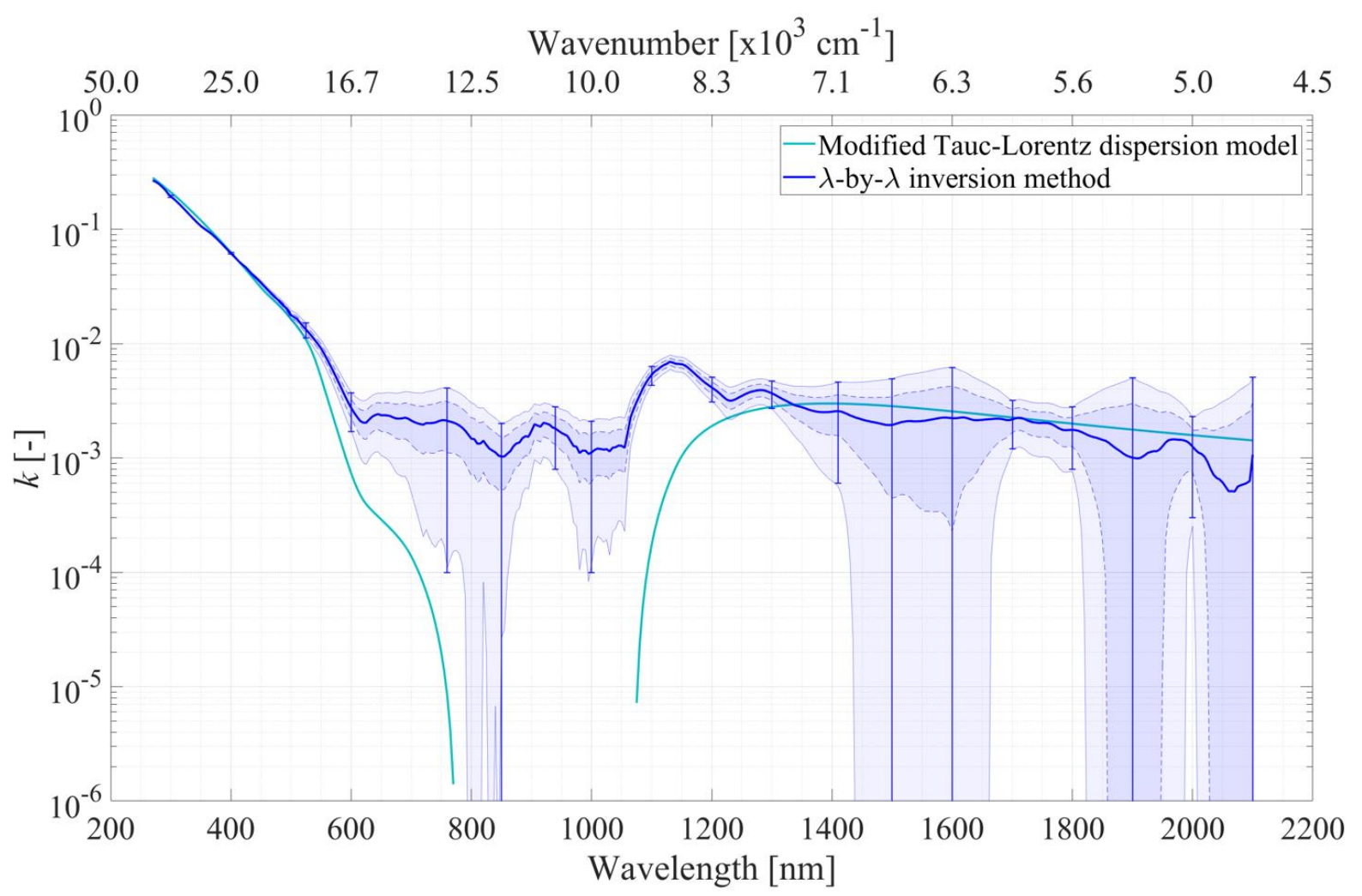

359

360

361

362

363

364

365

366

367

368

369

370

371

372

373

374

375

376

Figure 3: Imaginary part $k$ of the complex refractive index of Pluto aerosol analogues $\mathrm{P}_{\mathrm{H}}$ determined by spectroscopic ellipsometry with a modified Tauc-Lorentz dispersion model (in turquoise blue) and with a " $\lambda$-by- $\lambda$ " numerical inversion method (in dark blue). The $2 \sigma$ error bars were experimentally determined for the " $\lambda$-by- $\lambda$ " inversion method at different wavelengths. The uncertainties were then linearly interpolated between two error bars in order to obtain the envelope of $2 \sigma$ uncertainty (in light blue). The envelope determined by dashed lines corresponds to $1 \sigma$ uncertainty. Note that the $y$-axis was cropped at $10^{-6}$, while the $2 \sigma$ uncertainties of $k$-values reach 0 in the [800-850], [1450-1650] and [1820-2100] nm regions.

In Figure 2, the dark-blue and turquoise-blue curves present the same profile, with a maximal $n$-value of about 1.77 at $325-330 \mathrm{~nm}$ and decreasing values of $n$ index between 330 and $2100 \mathrm{~nm}$. In Figure 3, $k$-values determined with the two models are very similar in the [270490] and [1150-2100] $\mathrm{nm}$ regions. The dark-blue and turquoise-blue curves share the same slope, indicating similar values for the bandgap energy (also called "absorption edge", i.e. the wavelength at which there is a sharp increase of the $k$ index). The bandgap energy determined with the modified Tauc-Lorentz dispersion model (turquoise-blue curve) is around $575 \mathrm{~nm}$, while it is around $595 \mathrm{~nm}$ with the " $\lambda$-by- $\lambda$ " numerical inversion method (dark-blue curve). Beyond the bandgap energy, the behaviour of the $k$ indices is the same, reaching an asymptotic value of $\sim 2 \times 10^{-3}$ between 600 and $2100 \mathrm{~nm}$. 
377 From Figures 2 and 3, we can conclude that the choice of the fitting model - modified Tauc-

Lorentz or " $\lambda$-by- $\lambda$ " - has no significant impact on the $n$ and $k$ indices determined for $\mathrm{P}_{\mathrm{H}}$ sample in the [270-600] and [1150-2100] nm regions. Each method has advantages and drawbacks (specified in Sections II.3.a, II.3.b, and II.3.c). In this work, while the modified Tauc-Lorentz dispersion model was adapted for the determination of the thin-films thickness, it could not manage to determine non-null values for $k$ indices between 740 and $1105 \mathrm{~nm}$. This is due to the software used, which cannot compute $k$-values below $10^{-4}$. Therefore, we list in Tables SI.1, SI.2 and SI.3 (found in Supplementary Information) the optical constants of Pluto aerosol analogues $\mathrm{P}_{\mathrm{H}}, \mathrm{P}_{400}$ and $\mathrm{P}_{650}$ obtained by spectroscopic ellipsometry and determined with the " $\lambda$ by- $\lambda$ " fitting method from 270 to $2100 \mathrm{~nm}$ with a $5 \mathrm{~nm}$ increment. Note that $n$ and $k$ indices presented in all the figures of the manuscript and listed in Tables SI.1-3 were smoothed by a moving mean with a step of 15 data points. All the subsequent figures display the smoothed optical constants determined with the " $\lambda$-by- $\lambda$ " numerical inversion method.

\section{Impact of the altitude (or epoch) of aerosol formation on the optical constants and implications for radiative transfer}

$\mathrm{P}_{\mathrm{H}}, \mathrm{P}_{400}$ and $\mathrm{P}_{650}$ samples were produced with different $\mathrm{N}_{2}: \mathrm{CH}_{4}: \mathrm{CO}$ ratios. As $\mathrm{CH}_{4}$ mixing ratio strongly varies throughout the atmospheric column (Young et al. 2018), the purpose of comparing these three samples is to determine if the altitude of formation of the aerosols in Pluto's atmosphere has an impact on their optical constants.

\section{Effect on real part $n$ of the complex refractive index}

Figures 4 shows the real part $n$ of the complex refractive index of $\mathrm{P}_{\mathrm{H}}, \mathrm{P}_{400}$ and $\mathrm{P}_{650}$ samples as a function of wavelength. In this figure, the blue curve corresponds to $\mathrm{P}_{\mathrm{H}}$ sample, the red curve to $\mathrm{P}_{400}$ sample and the green curve to $\mathrm{P}_{650}$ sample. The $2 \sigma$ error bars (see Table SI.4 in Supplementary Information) were calculated based on the uncertainties of $\mathrm{I}_{s}^{\text {exp }}$ and $\mathrm{I}_{c}^{\text {exp }}$ for different wavelengths. The uncertainties were then linearly interpolated between two error bars in order to obtain the envelopes of uncertainty (in light blue, light red and light green). 


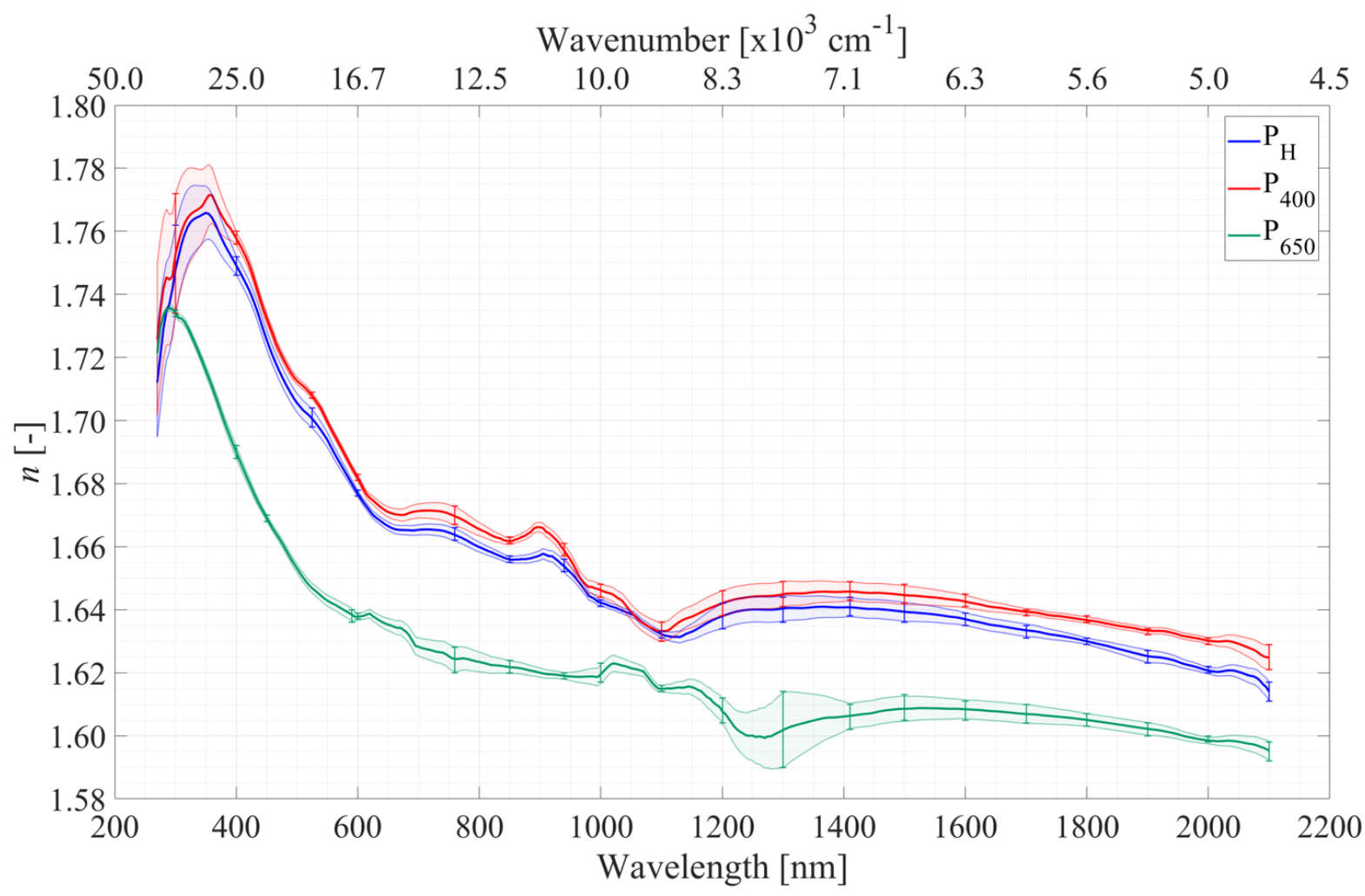

403

404

405

406

407

408

409

410

411

412

413

414

415

416

417

418

419

420

421

Figure 4: Real part $n$ of the complex refractive index of Pluto tholins determined by spectroscopic ellipsometry with a " $\lambda$-by- $\lambda$ " numerical inversion method. The blue, red and green curves respectively correspond to the samples $\mathrm{P}_{\mathrm{H}}, \mathrm{P}_{400}$ and $\mathrm{P}_{650}$. The $2 \sigma$ error bars were experimentally determined for different wavelengths and were then linearly interpolated in-between in order to obtain the envelopes of $2 \sigma$ uncertainty (in light blue, light red and light green). Note that the small variations observed in the $n$ curves at wavelengths above $600 \mathrm{~nm}$ should be considered with caution.

In Figure 4, we observe that the values of $n$ indices of $\mathrm{P}_{\mathrm{H}}$ and $\mathrm{P}_{400}$ samples are relatively similar, but substantially different from those of $\mathrm{P}_{650}$ sample. For $\mathrm{P}_{\mathrm{H}}$ and $\mathrm{P}_{400}$ samples, $n$-values increase from 1.71-1.73 to $\sim 1.77$ between 270 and $330 \mathrm{~nm}$. Then, $n$-values decrease with a steep slope from $\sim 1.77$ to around 1.67 between 330 and $650 \mathrm{~nm}$. The slope attenuates between 650 and $1100 \mathrm{~nm}$. Finally, the $n$ indices of $\mathrm{P}_{\mathrm{H}}$ and $\mathrm{P}_{400}$ reach an asymptotic value of 1.62-1.63 between 1100 and $2100 \mathrm{~nm}$. For $\mathrm{P}_{650}$ sample, $n$ index increases from 1.72 to around 1.74 between 270 and $305 \mathrm{~nm}$, followed by a steep decrease from around 1.74 to 1.64 between 305 and $600 \mathrm{~nm}$. The decreasing slope then attenuates between 600 and $1200 \mathrm{~nm}$, before $n$ index reaches an asymptotic value of 1.60-1.61 between 1200 and $2100 \mathrm{~nm}$. In summary, the increase of the $\mathrm{CH}_{4}: \mathrm{N}_{2}$ mixing ratio in the reactive gas mixture producing Pluto tholins leads to a decrease of $n$ index over the entire spectral range considered. As well, the maximum of $n$-value is shifted towards shorter wavelengths. 
The bell-shaped appearance between 270 and $400 \mathrm{~nm}$, although not observed in the studies of optical constants of Titan aerosol analogues (Brassé et al. 2015), appears to be a consistent trend in Pluto tholins, whatever inversion model - " $\lambda$-by- $\lambda$ " or modified Tauc-Lorentz - is used. In contrast, the small variations observed in the $n$-curves at wavelengths above $600 \mathrm{~nm}$ should be considered with caution. Indeed, we cannot totally rule out the fact that these small variations are introduced by the $n$ index being a function of wavelength $\lambda$ (Mistrik et al. 2017; Moise et al. 2015). Nevertheless, this dependence is especially observed on a large scale: when $\lambda$ increases, the $n$ index increases or decreases monotonically. In addition, in the literature concerning the optical constants of terrestrial aerosols or tholins materials (e.g., Cechalova et al. 2019; Khare et al. 1984; Liu et al. 2015; Mahjoub et al. 2012; Ramírez et al. 2002; SciammaO'Brien et al. 2012), the $n$ indices are often represented as a smooth profile, suggesting that there may be no physical reason to observe fine-scale variations of $n$ index.

The overall decreasing trend of $n$ indices from UV to near-IR was also observed in the studies of optical constants of Titan tholins, regardless of the production mode (type of energy source, gas pressure, gas temperature, $\mathrm{CH}_{4}: \mathrm{N}_{2}$ mixing ratios...) or the type of measurement (spectroscopic ellipsometry, spectrophotometry...) (Brassé et al. 2015; Imanaka et al. 2004; Khare et al. 1984; Mahjoub et al. 2012, 2014; Ramírez et al. 2002; Sciamma-O’Brien et al. 2012; Tran et al. 2003). In the considered wavelength range, $n$ indices of Pluto aerosol analogues vary from around 1.60 to 1.78 . These values are similar to the $n$-values determined for Titan aerosol analogues (Brassé et al. 2015). It thus seems that Pluto tholins, produced in gas mixtures composed of $\mathrm{N}_{2}, \mathrm{CH}_{4}$ and $\mathrm{CO}$, share first-order similar optical properties with Titan tholins. This similarity also extends to the chemical composition. Indeed, Jovanović et al. (2020) studied the chemical composition of Pluto aerosol analogues. They showed that the molecules constituting the tholins are composed of a repetition of a $\left(\mathrm{CH}_{2}\right)_{\mathrm{w}}(\mathrm{HCN})_{\mathrm{z}}$ random pattern, such as molecules in Titan aerosol analogues (Gautier et al. 2017; Maillard et al. 2018; Pernot et al. 2010), with, however, a significant incorporation of oxygen atoms. van Krevelen and te Nijenhuis (2009) reported that such $n$-values can also correspond to organic polymers including polyamides $-\mathrm{C}(=\mathrm{O})-\mathrm{NH}-$ and polyethers $R-\mathrm{O}-R^{\prime}$.

We also note in Figure 4 that the $n$ indices are higher for $\mathrm{P}_{\mathrm{H}}$ and $\mathrm{P}_{400}$ samples compared to the $\mathrm{P}_{650}$ one, with a systematic difference of about 0.05 over the entire wavelength range. This difference between $\mathrm{P}_{\mathrm{H}} / \mathrm{P}_{400}$ and $\mathrm{P}_{650}$ is significant and beyond the uncertainties determined for the three samples at different wavelengths. Regarding the effect of $\mathrm{CH}_{4}: \mathrm{N}_{2}$ mixing ratio on $n$ values, our results differ from those of Mahjoub et al. (2012). In this study concerning the 
influence of $\mathrm{CH}_{4}$ concentration on the optical constants of Titan aerosol analogues, the higher the $\mathrm{CH}_{4}: \mathrm{N}_{2}$ mixing ratio, the higher the $n$-values. In the case of Pluto tholins, the relation is reversed, suggesting an increasing influence of $\mathrm{CO}$ at lower $\mathrm{CH}_{4}$ concentration.

Aerosols formed in Pluto's lower atmosphere $(\leq 400 \mathrm{~km}$ of altitude) are thus expected to scatter and polarize the light differently than the aerosols formed at higher altitudes $(>400 \mathrm{~km}$ of altitude), due to their different $n$ indices (Boucher 2015; Kolokolova et al. 1997; Kolokolova \& Jockers 1997; Nakayama et al. 2018, 2012), and therefore differently affect the photon flux reaching the lower layers of the atmosphere and the surface.

\section{Effect on imaginary part $k$ of the complex refractive index}

Figure 5 shows the imaginary part $k$ of the complex refractive index computed for $\mathrm{P}_{\mathrm{H}}$ (top panel, blue curve), $\mathrm{P}_{400}$ (middle panel, red curve) and $\mathrm{P}_{650}$ (bottom panel, green curve) samples as a function of wavelength. The $2 \sigma$ error bars (see Table SI.4 in Supplementary Information) were calculated based on the uncertainties of $\mathrm{I}_{s}^{\text {exp }}$ and $\mathrm{I}_{c}^{\text {exp }}$ for different wavelengths. The uncertainties were then linearly interpolated in-between in order to obtain the envelopes of uncertainty (in light blue, light red and light green).

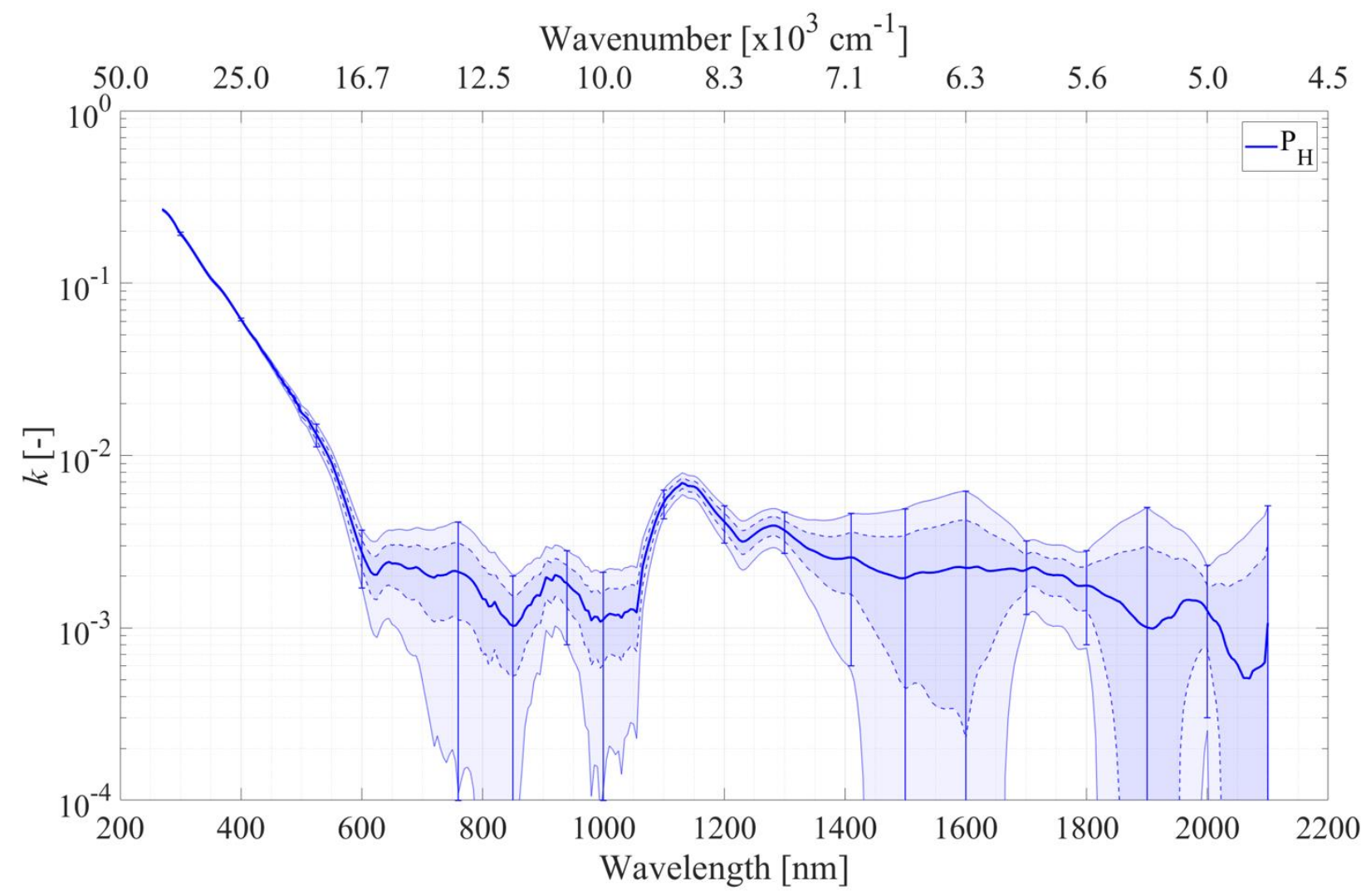




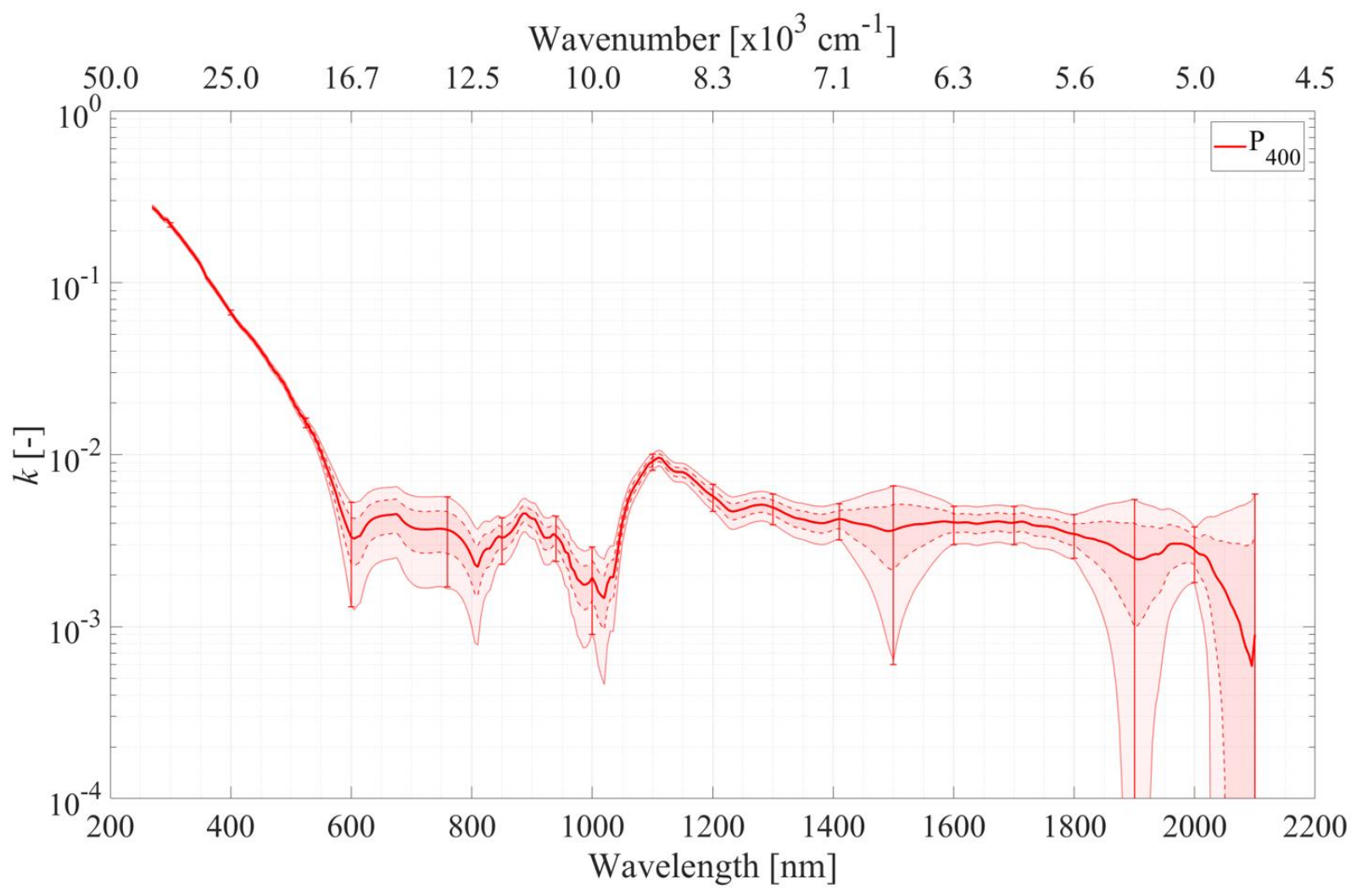

471

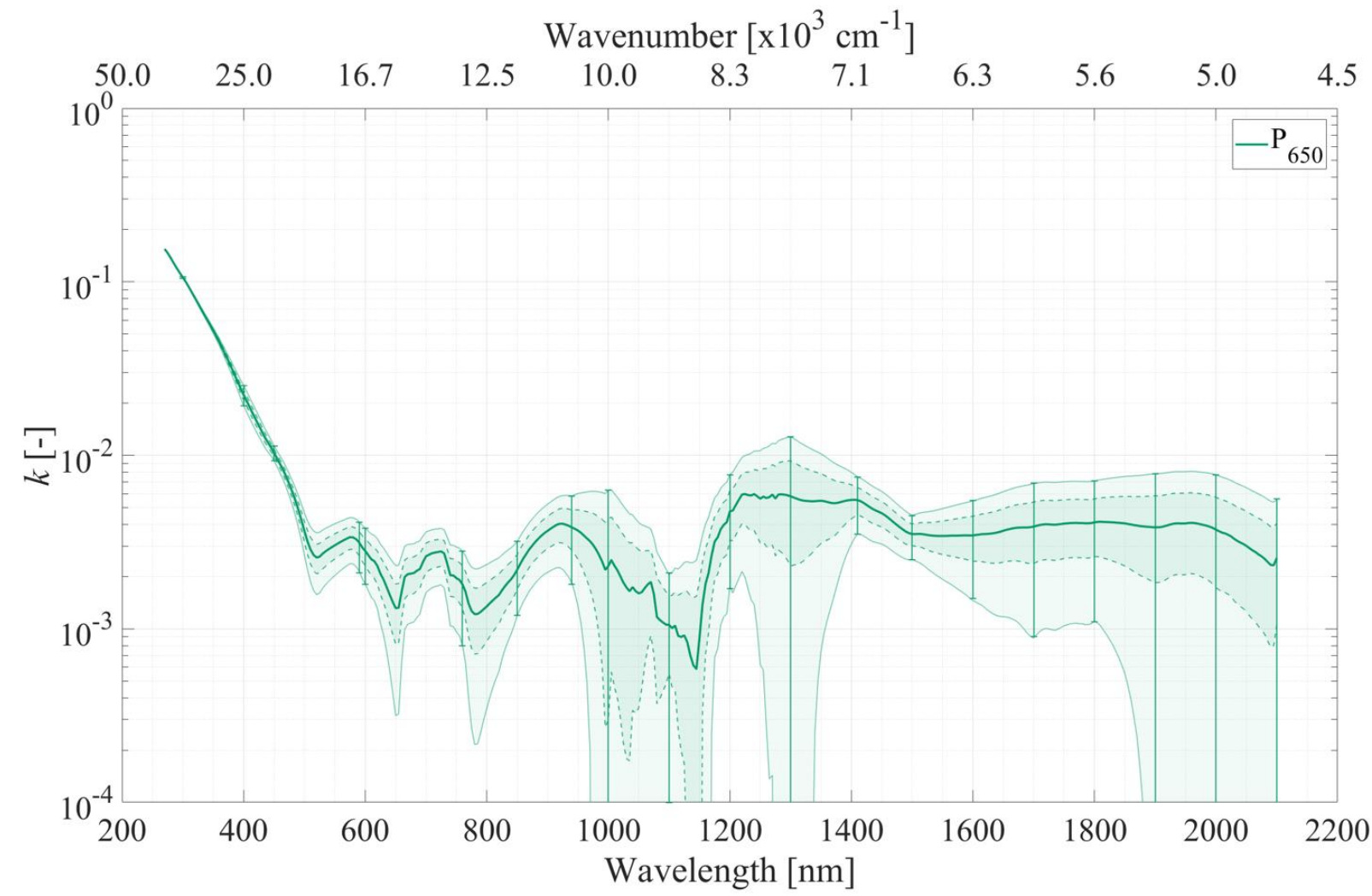

473 Figure 5: Imaginary part $k$ of the complex refractive index of Pluto tholins determined by spectroscopic

474 ellipsometry with a " $\lambda$-by- $\lambda$ " numerical inversion method. The blue (top panel), red (middle panel) and 475 green (bottom panel) curves respectively correspond to the samples $\mathrm{P}_{\mathrm{H}}, \mathrm{P}_{400}$ and $\mathrm{P}_{650}$. The $2 \sigma$ error bars 476 were experimentally determined for different wavelengths and were then linearly interpolated in- 
between in order to obtain the envelopes of $2 \sigma$ uncertainty (in light blue, light red and light green). The envelopes determined by dashed lines correspond to $1 \sigma$ uncertainty. The $y$-axis was cropped at $10^{-4}$. The $2 \sigma$ uncertainties of $k$-values reach 0 in the [790-850], [1450-1650] and [1820-2100] nm regions for $\mathrm{P}_{\mathrm{H}}$ sample, in the [1870-1930] and [2020-2100] nm regions for $\mathrm{P}_{400}$ sample and in the [980-1180], [1270$1330]$ and [1880-2100] nm regions for $\mathrm{P}_{650}$ sample. Note that the large variations observed in the $k$ curves at wavelengths above $600 \mathrm{~nm}$ should be considered with caution.

In Figure 5, we observe that the $k$-values of $\mathrm{P}_{\mathrm{H}}$ and $\mathrm{P}_{400}$ are significantly higher for wavelengths between 270 and $600 \mathrm{~nm}$ than those of $\mathrm{P}_{650}$. From 600 to $2100 \mathrm{~nm}$, the trend is less clear, because of larger uncertainties on $k$-values. For $\mathrm{P}_{\mathrm{H}}$ and $\mathrm{P}_{400}$ samples, $k$-values plummet from $2.7 \times 10^{-1}$ to around $4.5 \times 10^{-3}$ between 270 and $590 \mathrm{~nm}$. The bandgap energy is estimated to be around $590 \mathrm{~nm}$. For $\mathrm{P}_{650}$ sample, the $k$ index steeply decreases from $1.5 \times 10^{-1}$ down to around $2 \times 10^{-3}$ between 270 and $520 \mathrm{~nm}$. The bandgap energy is estimated at around $520 \mathrm{~nm}$. Due to the large uncertainties on $k$-values determined for the three samples above $600 \mathrm{~nm}$ and because this kind of material is weakly absorbent in the near-IR (Brassé et al. 2015), the large variations observed in the $k$-curves should be considered with caution. $k$ indices for all samples probably reach an asymptotic value of a few $10^{-3}$ between 600 and $2100 \mathrm{~nm}$.

The 2-steps behaviour of $k$-values - sharp decrease followed by a plateau - was also observed for Titan aerosol analogues in the considered wavelength range (Brassé et al. 2015; Imanaka et al. 2004; Khare et al. 1984; Mahjoub et al. 2012, 2014; Ramírez et al. 2002; Sciamma-O’Brien et al. 2012; Tran et al. 2003).

We do not have a definitive explanation for the variations observed beyond $600 \mathrm{~nm}$ in the $k$ curves of the 3 samples $\mathrm{P}_{\mathrm{H}}, \mathrm{P}_{400}$ and $\mathrm{P}_{650}$. Nevertheless, assuming that the variations observed are consistent absorption bands (see Table 2): (1) the absorption band around $725 \mathrm{~nm}$ observed in $\mathrm{P}_{650}$ sample could be ascribed to $\mathrm{C}-\mathrm{H}$ stretching $4^{\text {th }}$ overtone in $-\mathrm{CH}_{x}(x=1,2$ and 3$)$ and in Aromatic- $\mathrm{CH}$ and to $\mathrm{O}-\mathrm{H}$ stretching $3^{\text {rd }}$ overtone in $\mathrm{R}-\mathrm{OH}$ and in Aromatic- $\mathrm{OH}$; (2) the absorption band between 900 and $950 \mathrm{~nm}$ observed in all samples could be attributed to $\mathrm{C}-\mathrm{H}$ stretching $3^{\text {rd }}$ overtone and to $\mathrm{O}-\mathrm{H}$ stretching $2^{\text {nd }}$ overtone; (3) the absorption band between 1100 and $1150 \mathrm{~nm}$ observed in $\mathrm{P}_{\mathrm{H}}$ and $\mathrm{P}_{400}$ samples could correspond to $\mathrm{C}-\mathrm{H}$ stretching $2^{\text {nd }}$ overtone and to $-\mathrm{C}=\mathrm{O}$ stretching $4^{\text {th }}$ overtone; (4) the absorption band between 1950 and $2000 \mathrm{~nm}$ observed in $\mathrm{P}_{\mathrm{H}}$ and $\mathrm{P}_{400}$ samples could be in agreement with $-\mathrm{C}=\mathrm{O}$ stretching $2^{\text {nd }}$ overtone in $R-(\mathrm{C}=\mathrm{O})-R^{\prime}, R-(\mathrm{C}=\mathrm{O})-\mathrm{O} R^{\prime}$ and in $R-(\mathrm{C}=\mathrm{O})-\mathrm{NH}_{2}$ and to $\mathrm{N}-\mathrm{H}$ combination in $R-$ $(\mathrm{C}=\mathrm{O})-\mathrm{NH}_{2}$ and in aromatic amines (Stenberg et al. 2010; Workman \& Weyer 2008; Xiaobo 
et al. 2010). These overtones are conceivable, given the chemical composition determined for Pluto tholins by Jovanović et al. (2020).

Table 2: Overtone and combination near-IR band assignment of potential absorption bands found in the $k$ profiles presented in Figure 5. The band assignments are based on Stenberg et al. (2010), Workman \& Weyer (2008) and Xiaobo et al. (2010).

\begin{tabular}{|c|c|c|}
\hline Wavelength [nm] & Overtone and combination near-IR band assignment & $\begin{array}{l}\text { Present in } \\
\text { sample } \mathrm{P}_{x}\end{array}$ \\
\hline 725 & $\begin{array}{l}\text { - } \mathrm{C}-\mathrm{H} \text { stretching } 4^{\text {th }} \text { overtone in }-\mathrm{CH}_{x}(x=1,2 \text { and } \\
\text { 3) and in Aromatic-CH } \\
\text { - } \mathrm{O}-\mathrm{H} \text { stretching } 3^{\text {rd }} \text { overtone in } R-\mathrm{OH} \text { and in } \\
\text { Aromatic-OH }\end{array}$ & $\mathrm{P}_{650}$ \\
\hline $900-950$ & $\begin{array}{l}\text { - } \mathrm{C}-\mathrm{H} \text { stretching } 3^{\text {rd }} \text { overtone in }-\mathrm{CH}_{x}(x=1,2 \\
\text { and 3) and in Aromatic- } \mathrm{CH} \\
\text { - } \mathrm{O}-\mathrm{H} \text { stretching } 2^{\text {nd }} \text { overtone in } R-\mathrm{OH} \text { and in } \\
\text { Aromatic-OH }\end{array}$ & $\mathrm{P}_{\mathrm{H}}, \mathrm{P}_{400}, \mathrm{P}_{650}$ \\
\hline $1100-1150$ & $\begin{array}{l}\text { - } \mathrm{C}-\mathrm{H} \text { stretching } 2^{\text {nd }} \text { overtone in }-\mathrm{CH}_{x}(x=1,2 \\
\text { and } 3) \text { and in Aromatic- } \mathrm{CH} \\
\text { - }-\mathrm{C}=\mathrm{O} \text { stretching } 4^{\text {th }} \text { overtone in } R-(\mathrm{C}=\mathrm{O})-R^{\prime} \text {, } \\
R-(\mathrm{C}=\mathrm{O})-\mathrm{O} R^{\prime} \text { and in } R-(\mathrm{C}=\mathrm{O})-\mathrm{NH}_{2}\end{array}$ & $\mathrm{P}_{\mathrm{H}}, \mathrm{P}_{400}$ \\
\hline $1950-2000$ & $\begin{array}{c}-\mathrm{C}=\mathrm{O} \text { stretching } 2^{\text {nd }} \text { overtone in } R-(\mathrm{C}=\mathrm{O})-R^{\prime} \text {, } \\
R-(\mathrm{C}=\mathrm{O})-\mathrm{O} R^{\prime} \text { and in } R-(\mathrm{C}=\mathrm{O})-\mathrm{NH}_{2} \\
\text { - } \quad \mathrm{N}-\mathrm{H} \text { combination in } R-(\mathrm{C}=\mathrm{O})-\mathrm{NH}_{2} \text { and in } \\
\text { aromatic amines }\end{array}$ & $\mathrm{P}_{\mathrm{H}}, \mathrm{P}_{400}$ \\
\hline
\end{tabular}

From Figure 5, we can conclude that Pluto aerosol analogues significantly absorb UV and visible radiations, while their absorption is more moderate above $600 \mathrm{~nm}$. The strong absorption below 500-600 nm, in the blue-green spectral range, agrees with a brownish colour for Pluto tholins. This absorption is likely due to the presence of $\mathrm{N}$ - and O-bearing organic molecules with lone pair, such as $-\mathrm{NH},-\mathrm{NH}_{2},-\mathrm{NH} R, R-\mathrm{N}-R^{\prime},-\mathrm{NO}_{2},-\mathrm{OH},-\mathrm{COOH},-\mathrm{C}=\mathrm{O}, R-\mathrm{O}-R^{\prime}$ functional groups (Desyaterik et al. 2013; Imanaka et al. 2004; Liu et al. 2015; Mahjoub et al. 2012; Moise et al. 2015; Rao 1975). Due to the delocalization of $\pi$ electrons, fused aromatic rings, $\mathrm{N}$ - and $\mathrm{O}$-containing polycyclic aromatic compounds and unsaturated molecules with extensive conjugated multiple bonds also participate to the strong absorption of UV and visible 
wavelengths (D’Amico et al. 1980; Desyaterik et al. 2013; Imanaka et al. 2004; Lambe et al. 2013; Liu et al. 2015; Moise et al. 2015; Rao 1975; Tran et al. 2003; Zhong et al. 2012). Most of these chemical functions were actually detected in Pluto aerosol analogues by Jovanović et al. (2020), who showed the presence of unsaturated N- and O-bearing molecules in large amount.

When $\mathrm{CH}_{4}$ concentration increases, the bandgap energy shifts towards shorter wavelengths. $k$ index thus depends on the $\mathrm{CH}_{4}: \mathrm{N}_{2}$ mixing ratio used to produce the tholins. Indeed, from 270 to around $600 \mathrm{~nm}$, where the uncertainties are low, we can see that $\mathrm{P}_{\mathrm{H}}$ and $\mathrm{P}_{400}$ samples, produced with a lower $\mathrm{CH}_{4}$ concentration, present higher $k$-values than $\mathrm{P}_{650}$ sample, produced with a higher $\mathrm{CH}_{4}$ concentration.

This anti-correlation between $k$-values and $\mathrm{CH}_{4}: \mathrm{N}_{2}$ mixing ratio was also detected by Mahjoub et al. (2012) in their study concerning Titan tholins. They attributed this anti-correlation to the proportion of N-bearing molecules: when $\mathrm{CH}_{4}$ concentration decreases, N-containing molecules increase in proportion and contribute to a higher absorption of UV and visible radiations (Mahjoub et al. 2012, 2014). This conclusion was also proposed by Imanaka et al. (2004), when comparing their Titan tholins with Uranus/Neptune tholins from Khare et al. (1987) study, produced in $\mathrm{CH}_{4}: \mathrm{H}_{2}$ gas mixtures. They hypothesized that $\mathrm{N}$-incorporation in Titan tholins could increase $k$-values in the UV-Vis spectral range, in agreement with the laboratory experiments realized by Scattergood and Owen (1977). Indeed, nitrogen promotes unsaturation. When $\mathrm{N}_{2}$ content is increased in the reactive gas mixture forming Pluto tholins (case of $\mathrm{P}_{\mathrm{H}}$ and $\mathrm{P}_{400}$ samples), the formation of $\mathrm{sp}^{2}$ bonds is favoured. In contrast, when the reactive gas mixture contains more $\mathrm{CH}_{4}$ (case of $\mathrm{P}_{650}$ sample), the presence of large amounts of hydrogen promotes the formation of $\mathrm{sp}^{3}$ bonds, which have no effect on $\pi-\pi^{*}$ electronic transitions in UV and visible wavelength ranges (Ferrari et al. 2003; Quirico et al. 2008).

Another explanation to both higher $n$ - and $k$-values for $\mathrm{P}_{\mathrm{H}}$ and $\mathrm{P}_{400}$ could be the higher oxidation of these samples. Indeed, Jovanović et al. (2020) showed that $\mathrm{P}_{400}$ sample contains more oxygen in mass than $\mathrm{P}_{650}$. Studies realized on optical properties of terrestrial secondary organic aerosols (Cappa et al. 2011; Lambe et al. 2013; Moise et al. 2015; Nakayama et al. 2018, 2012) and of oxidized Early Earth- and Exoplanets-like aerosols (Gavilan et al. 2017) showed that $n$ indices increase when the oxidation level of the aerosols increases. Moreover, the more the aerosols are oxidized, the more they absorb UV and visible radiations. 
We can thus expect that aerosols formed at different altitudes in Pluto's atmosphere will differently absorb radiations reaching the atmosphere. In particular, Pluto's aerosols formed at or below $400 \mathrm{~km}$ of altitude above the surface, due to their higher $\mathrm{N}$ - and $\mathrm{O}$-content, may absorb more UV and visible wavelengths than aerosols formed above $400 \mathrm{~km}$ of altitude.

Besides, as mentioned in Section II.1, the samples studied here could also be considered as analogues of aerosols produced during different seasons or epochs of Pluto (Bertrand et al. 2019; Bertrand \& Forget 2016; Stern et al. 2017a). In Bertrand and Forget (2016), their Pluto General Circulation Model (GCM) predicted a mean $\mathrm{CH}_{4}$ atmospheric mixing ratio in 2200 (terrestrial year; northern winter/southern summer on Pluto) 5 times higher than that observed in 2015. This is due to the condensation of $\mathrm{N}_{2}$ onto the surface, which also leads to a decrease in surface pressure as low as $10^{-5}-10^{-6}$ mbar; the atmosphere being thinner, the methane is less diluted (Bertrand \& Forget 2016; Stern et al. 2017a). However, considering that the pressure in the atmosphere would still be sufficient to allow the formation of haze particles (Johnson et al. 2021), one would expect less absorbent aerosols, such as $\mathrm{P}_{650}$ tholins, differently affecting the thermal profile of Pluto and the photon flux reaching the surface.

\section{New input parameters for Pluto atmospheric and surface models}

In this section, we first compare the optical constants determined in this study with those from the study by Khare et al. (1984). Then, we discuss the application of our Pluto tholins optical constants to the modelling of Pluto's surface and atmosphere.

\section{Comparison of our optical constants with those of Titan tholins from the study by Khare et al. (1984)}

Khare et al. (1984) did a pioneer work to determine the optical constants $n$ and $k$ of Titan aerosol analogues from soft X-ray to microwave frequencies. For this purpose, they combined transmittance, specular reflectance, interferometric, Brewster angle and ellipsometric polarization measurements on thin films synthesized on various substrates. Although the large considered wavelength range is valuable, the employed method - combination of different thin films and different measurement techniques, with interpolations - induces major uncertainties. Moreover, Brassé et al. (2015) reviewed why Khare et al. (1984) optical constants are not representative of Titan's aerosols at different wavelengths.

Even though several other teams have since then determined optical properties of Titan aerosol analogues (Imanaka et al. 2012; Mahjoub et al. 2012, 2014; Ramírez et al. 2002; Sciamma- 
O'Brien et al. 2012; Tran et al. 2003; Vuitton et al. 2009), optical constants from Khare et al. (1984) are still widely used by the modelling teams to explain atmospheric and surface properties of Titan, often by introducing ad hoc modifications to them to fit the observations better (Brossier et al. 2018; Fan et al. 2019; Griffith et al. 2012; Lavvas et al. 2009; Liang et al. 2007; Lopes et al. 2016; Seignovert et al. 2017), but also of Pluto (Cheng et al. 2017; Grundy et al. 2018; Protopapa et al. 2017, 2020; Young et al. 2018; Zhang et al. 2017). This is partially due to the lack of optical constants of tholins-like materials covering a wide wavelength range.

Only the data by Khare et al. (1984) and Tran et al. (2003) for Titan tholins cover the same wavelength range as the Pluto optical constants determined in this work, and therefore are used for comparison purposes. In Figures 6 and 7, we respectively compare the $n$ index and the $k$ index of Pluto aerosol analogues $\mathrm{P}_{400}$ (red asterisks) to Khare et al. (1984) data (black asterisks), from 270 to $2100 \mathrm{~nm}$. Note that Titan tholins from Khare et al. (1984) were produced in a reactive gas mixture composed of $90 \%$ of $\mathrm{N}_{2}$ and $10 \%$ of $\mathrm{CH}_{4}$.

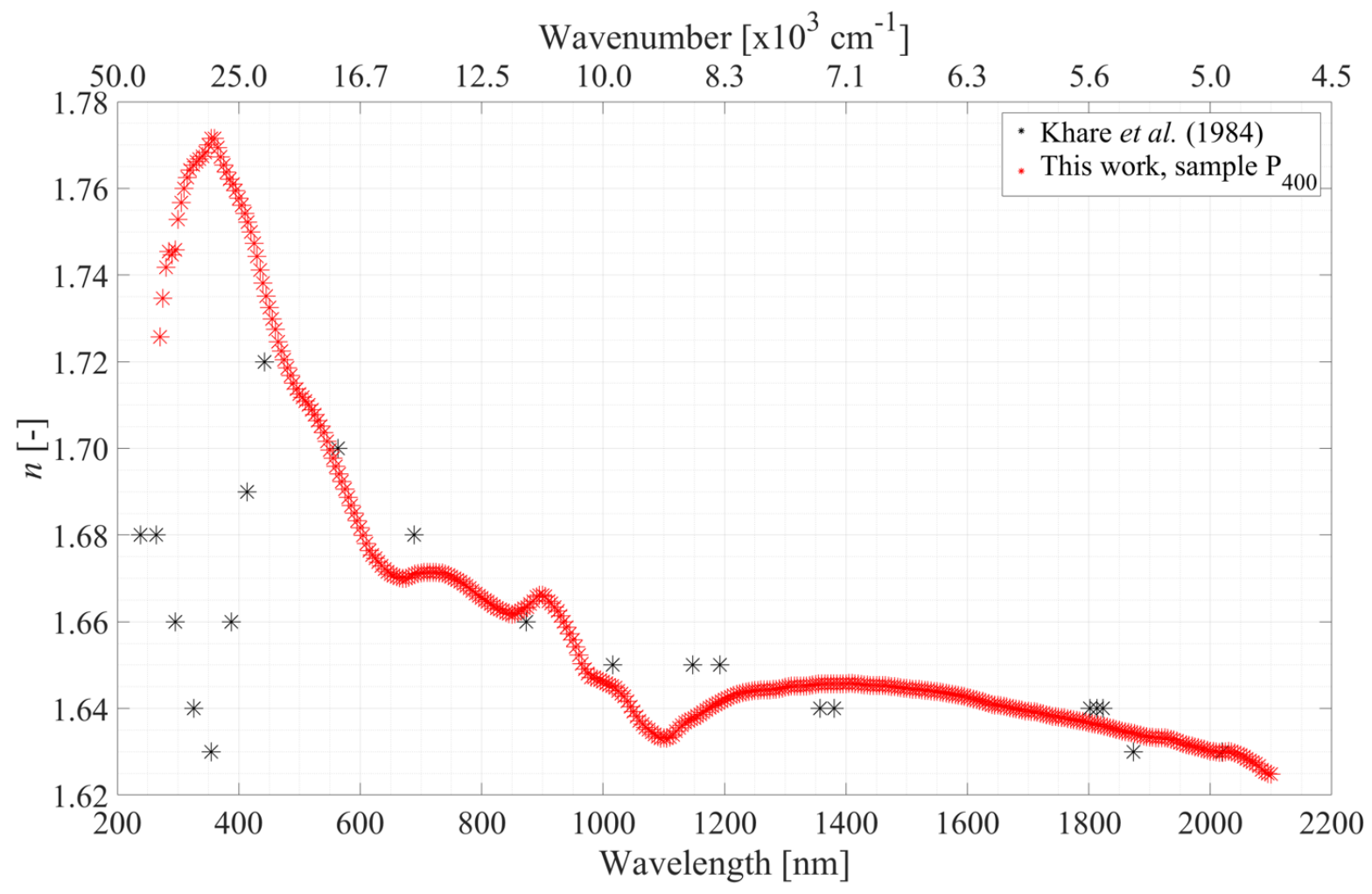

Figure 6: Comparison between the real part $n$ of the complex refractive index of our Pluto tholins $\mathrm{P}_{400}$ (red asterisks) and Titan tholins from Khare et al. (1984) study (black asterisks). 


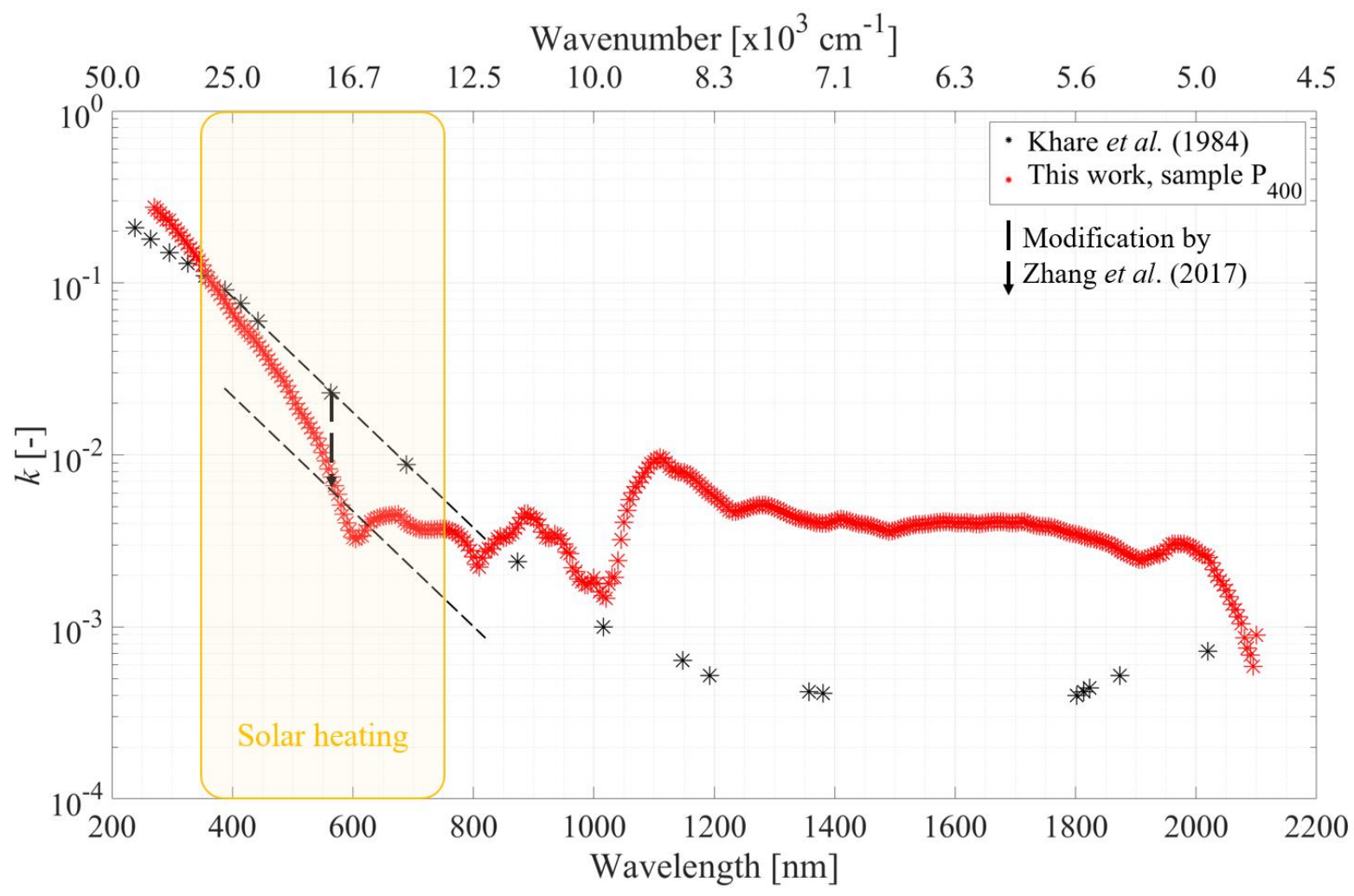

602

603

604

605

606

607

608

609

610

611

612

613

614

615

616

617

618

619

620

Figure 7: Comparison between the imaginary part $k$ the complex refractive index of our Pluto tholins $\mathrm{P}_{400}$ (red asterisks) and Titan tholins from Khare et al. (1984) study (black asterisks). The black dashed arrow indicates the modification implemented by Zhang et al. (2017) to the $k$-values from Khare et al. (1984) in the region where solar heating dominates (represented by the yellow rectangle) in order to adjust Pluto's thermal balance.

In Figure 6, we observe that the $n$ index of Pluto tholins $\mathrm{P}_{400}$ is similar to that of Khare et al. (1984) Titan tholins from 450 to $2100 \mathrm{~nm}$. Below $450 \mathrm{~nm}$, the behaviour is reversed. While $n$ value reaches a maximum near $330 \mathrm{~nm}$ for $\mathrm{P}_{400}$, Khare et al. (1984) data present a minimum $n$ value near $350 \mathrm{~nm}$. We do not have a definitive explanation for this behaviour of Khare et al. (1984) $n$ index, but it could be due to a low sampling combined with interpolation, a poor definition of the optical properties of the substrates used for the synthesis of the tholins thin films, or the light source used for the measurements being at the limit of its range.

Concerning the $k$ indices (Figure 7), the bandgap energy of Khare et al. (1984) tholins is shifted towards longer wavelengths (around $1200 \mathrm{~nm}$ ) compared to $\mathrm{P}_{400}$ sample (around $590 \mathrm{~nm}$ ). $k$ values of $\mathrm{P}_{400}$ are higher than those of Khare et al. (1984) below $380 \mathrm{~nm}$ and in the near-IR (> $900 \mathrm{~nm}$ ). These higher values can be explained by higher $\mathrm{N}$ - and O-content of Pluto aerosol analogues $\mathrm{P}_{400}$ (Cappa et al. 2011; Gavilan et al. 2017; Imanaka et al. 2004; Jovanović et al. 2020; Lambe et al. 2013; Mahjoub et al. 2012; Nakayama et al. 2018, 2012; Quirico et al. 2008). 
621 From 380 to around $800 \mathrm{~nm}, k$-values of $\mathrm{P}_{400}$ are lower than those of Khare et al. (1984), due to the shifted bandgap energy which is caused by higher $k$-values in the UV and a steeper slope in the UV-Vis for our $\mathrm{P}_{400}$ sample.

Even though $k$-values of $\mathrm{P}_{400}$ sample are one order of magnitude higher than those of Khare et al. (1984) between 1000 and $2000 \mathrm{~nm}$, they are constant and do not present the anomalous sharp increase beyond $1800 \mathrm{~nm}$ presented by the Khare et al. (1984) $k$-values. The fact that our $k$ values are one order of magnitude higher in the near-IR is consistent with published literature on Titan's aerosols. For example, Lavvas et al. (2010) and Larson et al. (2015) reported that the actual optical properties of Titan's aerosols should be about an order of magnitude larger in the near-IR compared to the optical constants from Khare et al. (1984).

\section{Application of our optical constants to Pluto's surface modelling}

Recently, Protopapa et al. (2020) studied the colouring materials across Pluto's surface. To support the conclusion developed by Grundy et al. (2018), Pluto's photochemical aerosols were considered as potential surface colouring agent. Thus, in their model, they implemented the $k$ indices determined for Titan tholins by Khare et al. (1984) and Tran et al. (2003). Nevertheless, in order to fit their models to spectra acquired in the regions of Cthulhu Macula and Lowell Regio by the imagers MVIC (Multispectral Visible Imaging Camera, a Vis-near-IR [400-975 nm] imager) and LEISA (Linear Etalon Imaging Spectral Array, an IR [1.25-2.5 $\mu \mathrm{m}$ ] spectral imager) constituting the Ralph instrument (Reuter et al. 2008), some modifications of $k$ indices by Khare et al. (1984) were necessary. Specifically, Protopapa et al. (2020): first applied no change to the $n$ index of Khare et al. (1984); secondly decreased the $k$-value of Khare et al. (1984) at $690 \mathrm{~nm}$ by $~ 55 \%$ to match the observations of Pluto in the RED filter between 540 and $700 \mathrm{~nm}$ (note that Protopapa et al. (2020) preserved the original sampling of Khare et al. (1984) explaining why the $k$-value was modified at $690 \mathrm{~nm}$ ); and thirdly assumed $k$ index to be constant between 1800 and $2200 \mathrm{~nm}$, given that the increase displayed by the $k$-values of Khare et al. (1984) beyond $1.8 \mu \mathrm{m}$ was not consistent with the observations.

Figure 8 was adapted from Protopapa et al. (2020). It displays the imaginary part $k$ of the complex refractive index (right $y$-axis) of our $\mathrm{P}_{400}$ sample (red curve, with an envelope of uncertainties represented in salmon pink), compared to those of the Titan tholins from the studies by Khare et al. (1984) (orange triangles) and Tran et al. (2003) (blue dots). Additionally, the set of $k$ indices determined by Protopapa et al. (2020) (black squares) that best fits the MVIC and LEISA New Horizons measurements acquired in the regions of Cthulhu Macula and Lowell 

to Protopapa et al. (2020) for details about the modelling of MVIC and LEISA spectra.

$\triangle$ Khare et al. (1984)

- Tran et al. (2003)

口 Protopapa et al. (2020), sample B

- This work, sample $P_{400}$

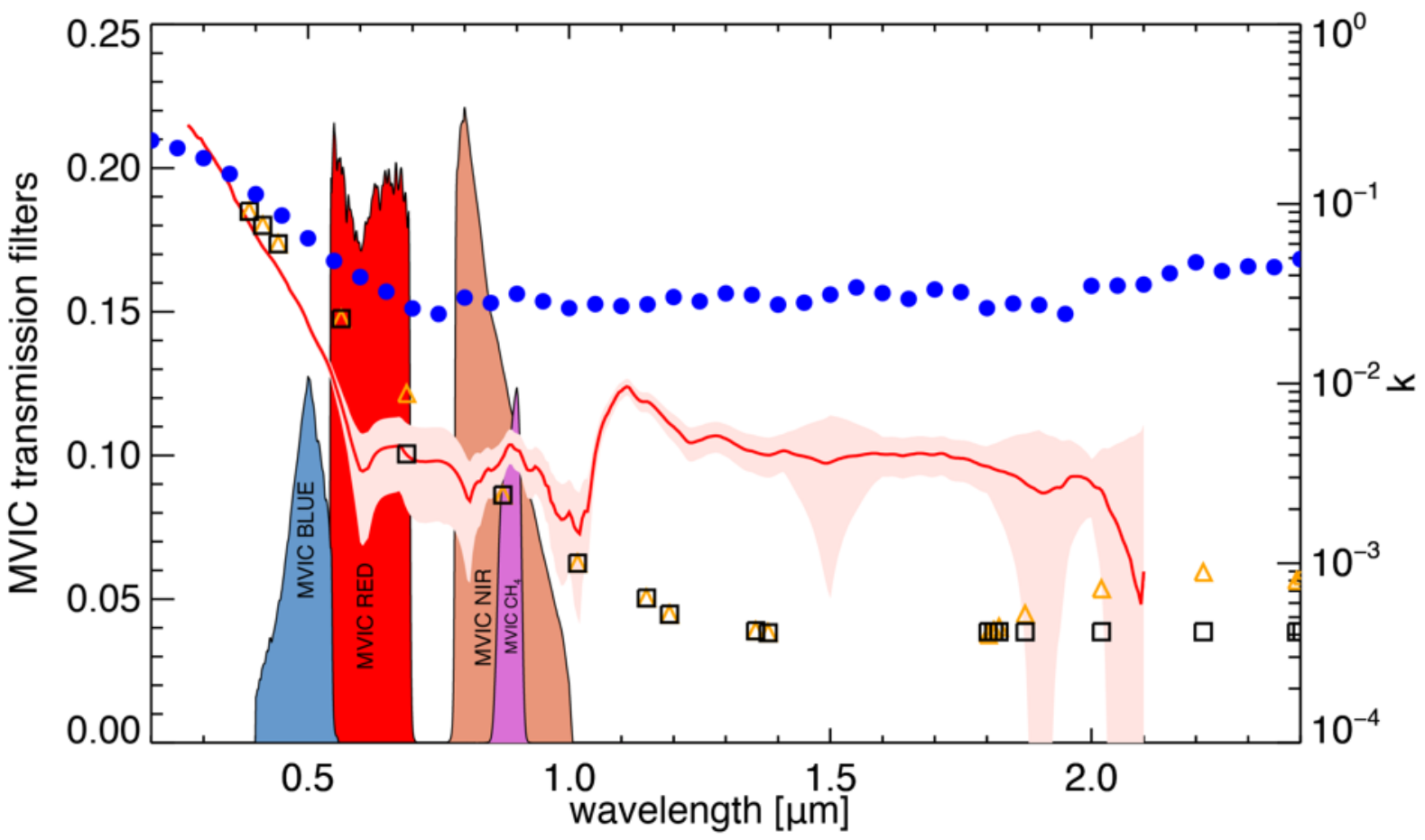

655

Figure 8: Figure adapted from Protopapa et al. (2020). Imaginary part $k$ of the complex refractive index (right $y$-axis) is represented as a function of wavelength. The red curve with the salmon-pink envelope corresponds to the $k$ index of our sample $\mathrm{P}_{400}$. The orange triangles correspond to the $k$ index determined by Khare et al. (1984), while the blue dots are from the study by Tran et al. (2003). The black squares are the $k$-values determined by Protopapa et al. (2020) that best fit the MVIC and LEISA data. The MVIC transmission filters (left $y$-axis) are shown for reference. The reader is referred to Protopapa et al. (2020) for details about the modelling.

Protopapa et al. (2020) recognized that the $k$-values of Titan tholins by Khare et al. (1984) present a sharp increase beyond $1.8 \mu \mathrm{m}$ (Figure 8, orange triangles), inconsistent with the Pluto New Horizons data. Therefore, Protopapa et al. (2020) modified the $k$-values by Khare et al. (1984) beyond $1.8 \mu \mathrm{m}$ assuming them to be constant with wavelengths up to $2.4 \mu \mathrm{m}$ and equal to the mean value computed between 1.35 and $1.45 \mu \mathrm{m}$ (see Figure 8, sample B from Protopapa et al. (2020)). This is consistent with our direct values for Pluto tholins $\mathrm{P}_{400}$. Indeed, as noted above, while the $k$-values of $\mathrm{P}_{400}$ sample are one order of magnitude higher than those of Khare 
et al. (1984) between 1000 and $2000 \mathrm{~nm}$, they are constant with wavelengths and do not display the sharp increase beyond $1.8 \mu \mathrm{m}$ presented by the Khare et al. (1984) $k$-values.

In Figure 8, we observe that the change to the Khare et al. (1984) data implemented at $690 \mathrm{~nm}$ by Protopapa et al. (2020) (black squares) to match the Pluto New Horizons observations is consistent with the $k$-curve of our Pluto tholins $\mathrm{P}_{400}$ (red curve, with the salmon-pink envelope). Also, sample B from Protopapa et al. (2020) shares the same slope in the UV-Vis as our $\mathrm{P}_{400}$ sample and is in agreement, within the uncertainties, with our data between 0.8 and $1.0 \mu \mathrm{m}$.

These considerations support the suitability of our optical constants to reproduce Pluto compositional observations at least in the UV and visible spectral ranges. Nevertheless, from 1.1 to $1.8 \mu \mathrm{m}$, our $k$-values are obviously larger by an order of magnitude with respect to the $k$ values adopted to reproduce Pluto New Horizons data by Protopapa et al. (2020). An in-depth modelling analysis of the Pluto New Horizons observations using as inputs our Pluto tholins optical constants throughout the full wavelength range, from 0.4 to $2.5 \mu \mathrm{m}$, is required to confirm that Pluto's colouration and composition can be attributed to the photochemical aerosols that we reproduced in our laboratory.

\section{Application of our optical constants to Pluto's atmosphere modelling}

In order to evaluate the effect of our Pluto tholins optical constants on aerosol properties, and to compare with those of Titan, we display the extinction coefficient $\mathrm{Q}_{\text {ext }}$ (Figure 9), the singlescattering albedo $\omega$ (Figure 10), and the absorption coefficient $Q_{\text {abs }}$ (Figure 11) for a spherical particle of $10 \mathrm{~nm}$ according to Mie theory (formalized in Bohren \& Huffman (1983)) and for a fractal aggregate of spheres (Rannou et al. 1997, 1999).

These parameters are key in the atmospheric radiative transfer (Bohren \& Huffman 1983). Qext is the ratio of the extinction cross section $\left(\sigma_{\mathrm{ext}}\right)$ and the geometric projection of the particle $\left(\sigma_{\mathrm{geo}}\right)$. It characterizes the extinction, i.e. the absorption and scattering of radiation by aerosols. $\omega$ represents the fraction of scattered radiation, while $(1-\omega)$ represents the fraction of absorbed radiation. $\omega$ is equal to 0 for a perfect absorber and equal to 1 for a perfect scatterer. These two coefficients control the amount of energy absorption by aerosols at solar wavelengths through $Q_{a b s}=(1-\omega) \times Q_{e x t}$ and the emission in thermal infrared $Q_{e m i}=Q_{a b s}=(1-\omega) \times Q_{e x t}$. They participate to the thermal balance of Pluto's atmosphere, as demonstrated by Zhang et al. (2017). 
At first glance, the scattering of haze at Pluto's limb in the forward direction indicates particles having radii in the range 0.1-0.5 $\mu \mathrm{m}$ (Gladstone et al. 2016; Stern et al. 2015). Further detailed analyses and arguments from microphysical models (Cheng et al. 2017; Gao et al. 2017; Zhang et al. 2017) yielded a better result with fractal aggregates of small grains. In these studies, a fractal dimension $D_{f}=2$ was assumed, the morphology of the haze particles is thus more like that of snowflakes instead of solid spheres. The study by Cheng et al. (2017) showed that spherical monomers should have $\sim 10 \mathrm{~nm}$ radius $\left(\mathrm{R}_{\mathrm{m}}\right)$ and aggregates should have a "bulk radius" (or "volume equivalent radius") of about $\mathrm{R}_{\mathrm{b}}=0.15 \mu \mathrm{m}$, corresponding to $\mathcal{N}=\left(\mathrm{R}_{\mathrm{b}} / \mathrm{R}_{\mathrm{m}}\right)^{3} \approx 3375$ monomers per aggregate. Such a fractal structure globally satisfies, although not perfectly, the main requirements to explain Pluto New Horizons observations (Cheng et al. 2017). This aerosol structure was also used by Zhang et al. (2017) to study Pluto's thermal balance. Note, however, that in the study by Gao et al. (2017), the particle size distributions of their model are displayed as a function of the "effective radius" $\mathrm{R}_{\mathrm{f}}$. Since their aerosols grow up to $\mathrm{R}_{\mathrm{f}}=0.15 \mu \mathrm{m}$, with a fractal dimension $\mathrm{D}_{\mathrm{f}}=2$, the corresponding aggregates appear much smaller $(\mathcal{N}=225)$ than those considered by Cheng et al. (2017), Zhang et al. (2017) and Lavvas et al. (2020) $(\mathcal{N}=3375)$. The most probable reason for this difference stands in that Cheng et al. (2017) and Lavvas et al. (2020) matched data from New Horizons containing information about the aerosol size, with observations made at different phase angles, while Gao et al. (2017) used data that are not sensitive to the aerosol size. Since aerosols are produced from growth mechanisms, they are expected to start as small spherical particles at high altitudes and continuously get larger by coagulation (direct gas-particle interactions) while sedimenting (Cheng et al. 2017; Gao et al. 2017; Zhang et al. 2017). Thus, both the properties of small spherical monomers and fractal aggregates are interesting.

In Figures 9 and 10, we compare, for several sets of optical constants, the parameters $Q_{\text {ext }}$ and $\omega$ computed for spherical monomers of $10 \mathrm{~nm}$ radius, distributed with a log-normal distribution and an effective variance $v$ of 0.03 (dotted lines), and for fractal aggregates of 3375 monomers of $10 \mathrm{~nm}$ radius (plain lines). We used the optical constants determined in this work $\left(\mathrm{P}_{\mathrm{H}}, \mathrm{P}_{400}\right.$ and $\mathrm{P}_{650}$, in blue, red and green respectively), optical constants determined for Titan tholins by Khare et al. (1984) (in black) and for Titan's aerosols from observations by Rannou et al. (2010) and Vinatier et al. (2012) (in pink). 


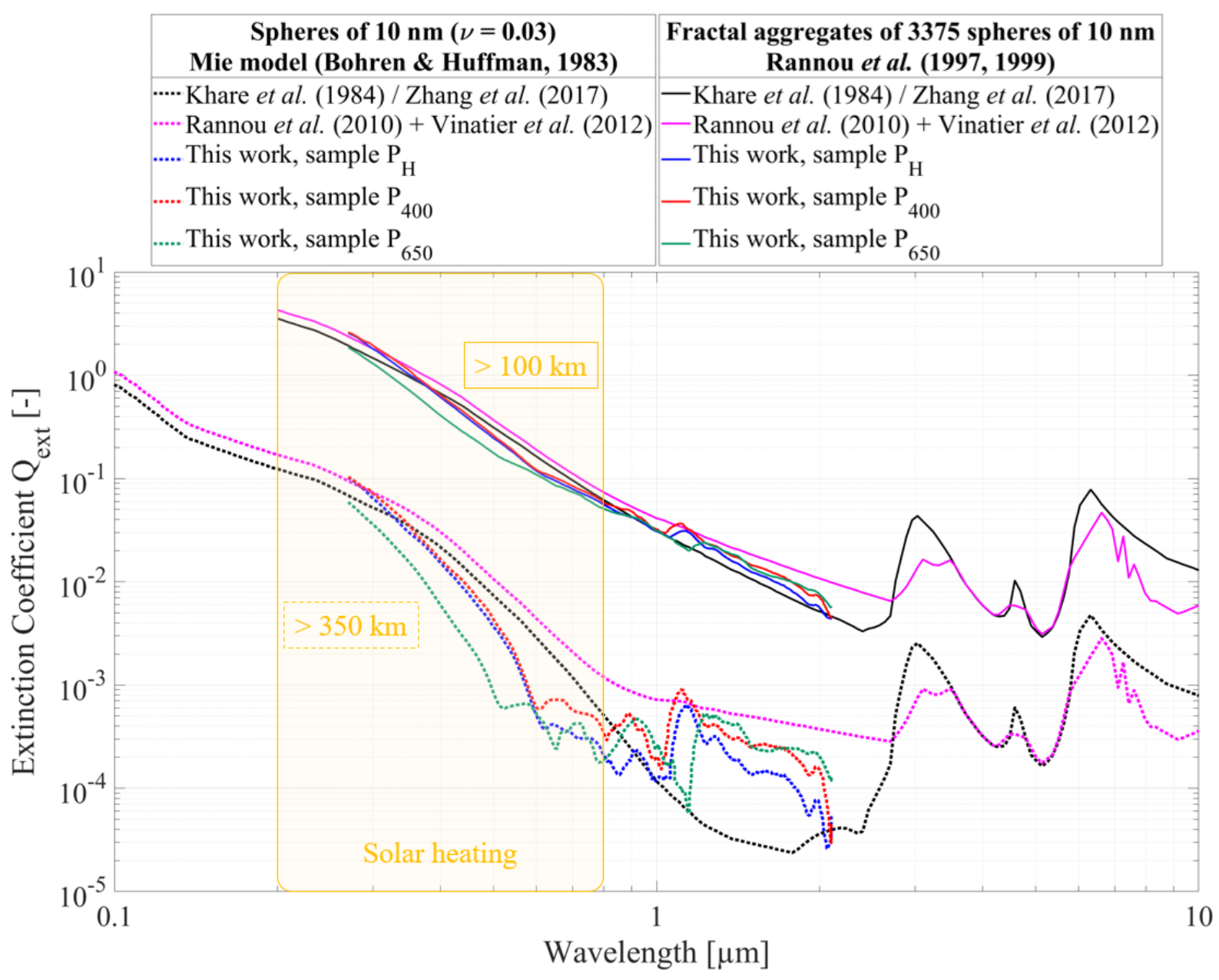

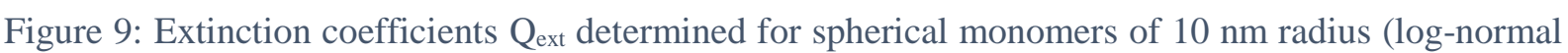

732 distribution, with effective variance $v=0.03$ ) with a Mie model formalized in Bohren \& Huffman (1983)

733 (dotted curves) and for fractal aggregates of 3375 monomers of $10 \mathrm{~nm}$ radius with the model developed

734 by Rannou et al. $(1997,1999)$ (plain curves). The black curves were generated with the optical constants

735 determined by Khare et al. (1984) for Titan tholins. Note that these Khare et al. (1984) optical constants

736 were adopted in Zhang et al. (2017) study. The pink curves were generated with the optical constants

737 determined by Rannou et al. (2010) and Vinatier et al. (2012) for Titan's aerosols. The blue, red and

738 green curves were generated with the optical constants determined respectively for our samples $\mathrm{P}_{\mathrm{H}}, \mathrm{P}_{400}$

739 and $\mathrm{P}_{650}$. The yellow rectangle indicates the region where solar heating dominates. The dotted curves

740 (spherical monomers) give information on the radiative transfer in the upper layers of Pluto's

741 atmosphere (> $350 \mathrm{~km}$ of altitude), while the plain curves (fractal aggregates) give information on the

742 radiative transfer in Pluto's lower atmosphere (between 100 and $300 \mathrm{~km}$ of altitude). 


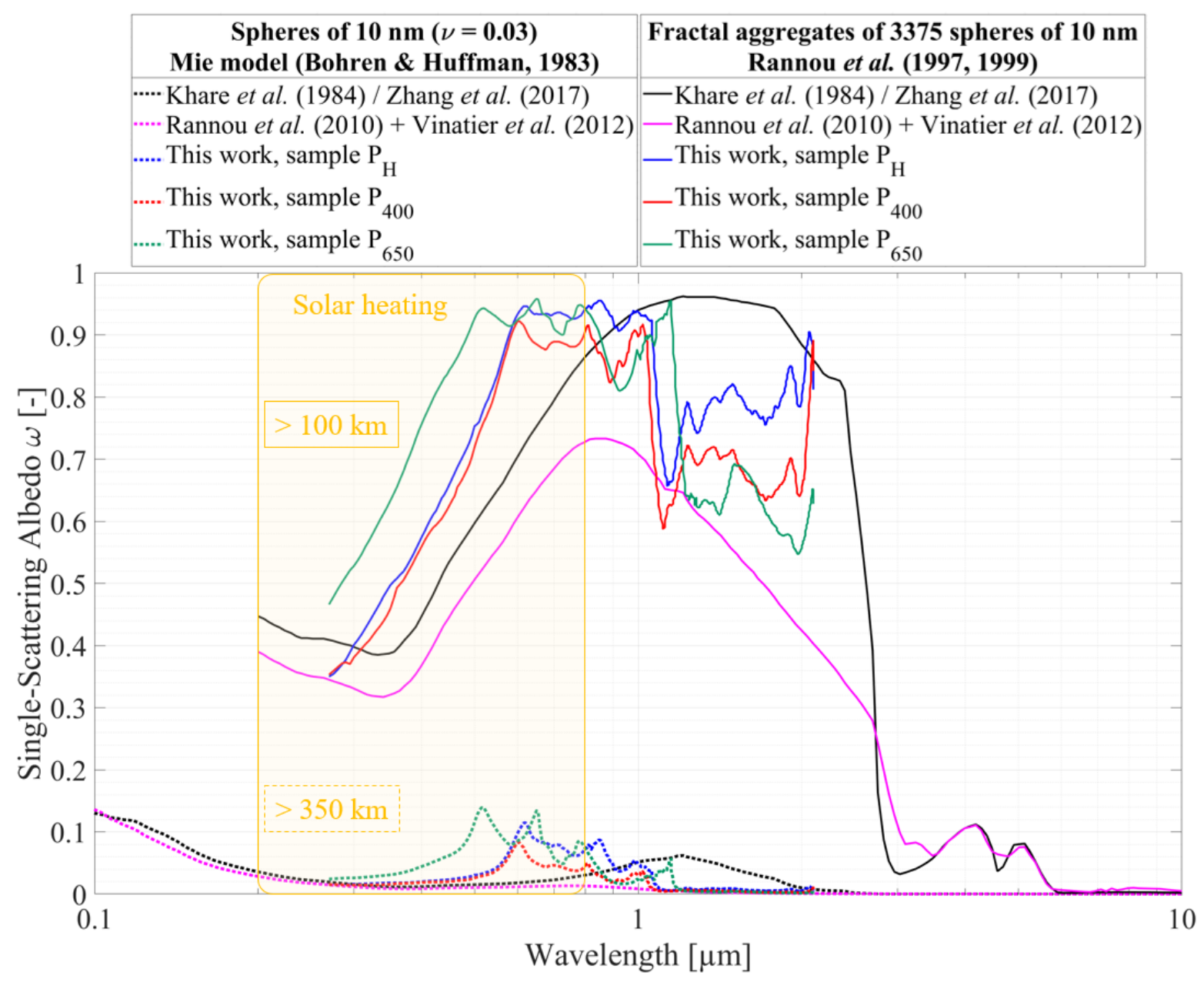

Figure 10: Same as Figure 9, but showing the single-scattering albedo $\omega$.

745 In Figure 9, we remark that the extinction coefficients $\mathrm{Q}_{\text {ext }}$ for spherical particles or fractal 746 aggregates computed with tholins relevant for Pluto have a steepest spectral slope at visible wavelengths (broadly from 0.2 to $0.8 \mu \mathrm{m}$, where most Pluto New Horizons photometrical data were taken, and where solar heating occurs) than those computed with optical constants relevant for Titan's aerosols.

In Figure 10, considering single spheres or fractal aggregates, we see that the single-scattering albedo $\omega$ is significantly higher in the UV and visible spectral ranges with the optical constants of Pluto tholins than with Titan optical constants. In the near-IR, $\omega$ of Pluto tholins are lower than that of Khare et al. (1984) Titan tholins. This suggests that Pluto's haze absorbs less in the UV-Vis wavelength range than Titan's haze.

However, to really assess the absorption efficiency of each type of particles, one has to compare the absorption coefficients $Q_{a b s}=(1-\omega) \times Q_{\text {ext }}$ (displayed in Figure 11). 


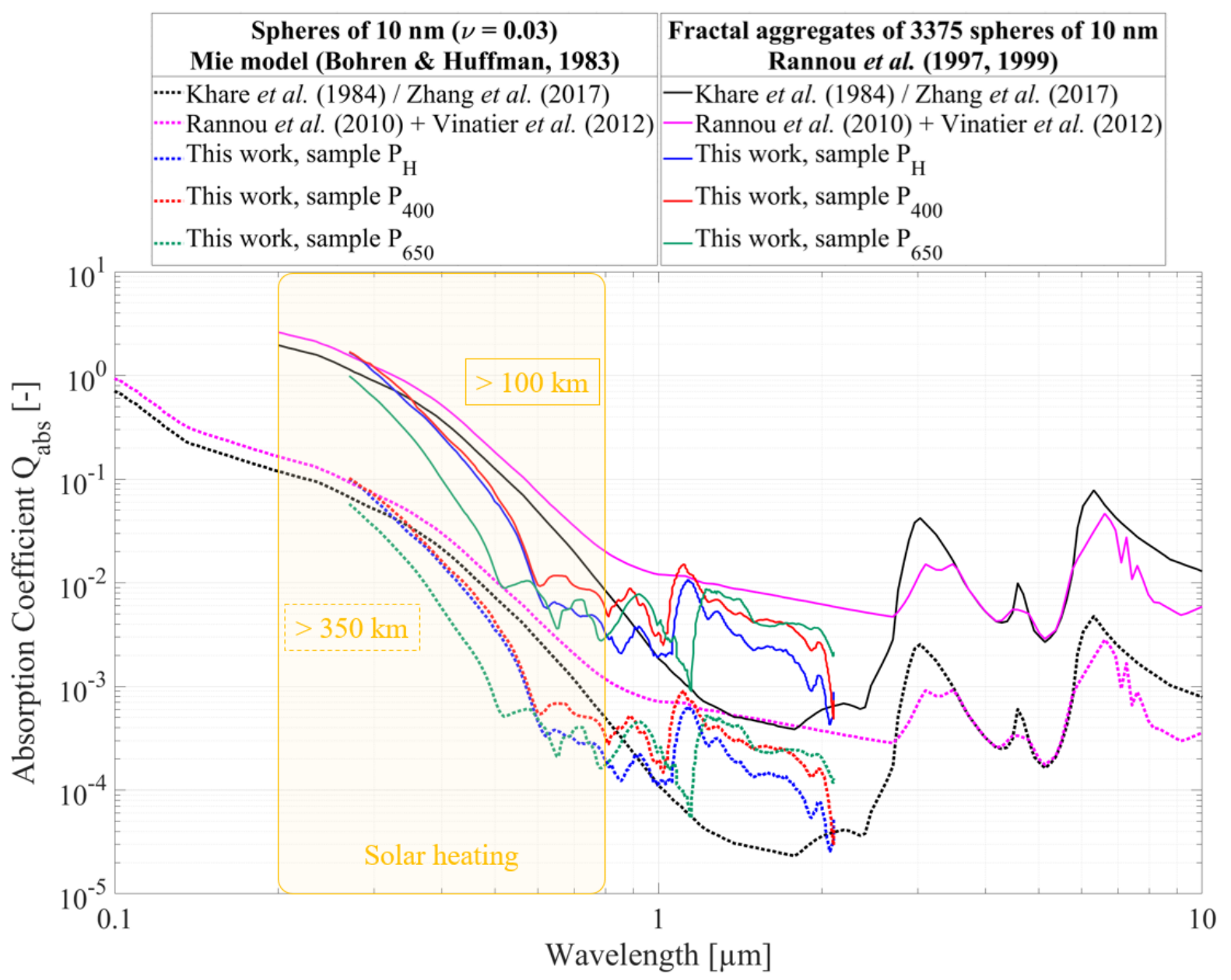

Figure 11: Same as Figure 9, but showing the absorption coefficients $\mathrm{Q}_{\text {abs. }}$

759

760

761

762

763

764

765

766

767

768

769

770

771

The absorption coefficients $Q_{\text {abs }}$ (Figure 11) computed with Pluto tholins optical constants are about equal to those computed with Titan optical constants at $0.3 \mu \mathrm{m}$. Nevertheless, they drop much faster with wavelengths and are lower by a factor 3 to 10 at wavelengths larger than $~ 0.4-$ $0.5 \mu \mathrm{m}$. This is true for small spherical particles and fractal aggregates.

Beyond $1 \mu \mathrm{m}$, the marked structures of Pluto tholins set the extinction coefficients Qext (Figure 9) between those obtained with optical constants of Khare et al. (1984) and Rannou et al. (2010), but this has less consequence on data analysis or on the thermal balance. On the other hand, the scattering efficiency $Q_{\text {sca }}=\omega \times Q_{\text {ext }}$, the phase functions and the asymmetry parameters are not modified at all by optical constants (see Supplementary Information, Figures A and B).

This has an important consequence: using other optical constants than those already used to analyse scattering photometry at Pluto's limb (Cheng et al. 2017; Gao et al. 2017; Gladstone et al. 2016; Stern et al. 2015) would not change the result about aerosol size and vertical profile. Nevertheless, optical constants of Pluto tholins presented in this study deeply modify the link 
between the scattering properties and the absorption properties, compared to Titan tholins. That is, for the same scattering efficiency, Pluto tholins absorb about 5 to 10 times less than Titan tholins at $0.5 \mu \mathrm{m}$ (effective wavelength of solar input).

To adjust Pluto's thermal balance, Zhang et al. (2017) used the optical constants from Khare et al. (1984), and had to decrease significantly (up to a factor 4) the absorption efficiency of aerosols in the visible wavelength range (see Figure 7, black dashed arrow), to increase it in thermal IR and grossly keep the same extinction properties to match Alice UV spectrometer retrieval for the haze extinction (Stern et al. 2015). Here we see that low absorption is a natural outcome of Pluto tholins produced for this study with chemical conditions representative of Pluto's atmosphere.

\section{Conclusion and Perspectives}

In this study, we determined the optical constants of Pluto aerosol analogues. Three types of tholins were produced from gas mixtures with different $\mathrm{N}_{2}: \mathrm{CH}_{4}: \mathrm{CO}$ proportions, in order to mimic aerosols formed at different altitudes in Pluto's current atmosphere. The sample $\mathrm{P}_{\mathrm{H}}$ is expected to be representative of aerosols formed below $350 \mathrm{~km}$ of altitude above the surface. The sample $\mathrm{P}_{400}$ is expected to mimic the aerosols formed at around $400 \mathrm{~km}$ of altitude. The sample $\mathrm{P}_{650}$ is expected to be analogous of aerosols formed between 600 and $700 \mathrm{~km}$ of altitude. These three gas mixtures differing in their $\mathrm{CH}_{4}: \mathrm{N}_{2}$ mixing ratio can also be considered as analogues of Pluto's atmosphere at different seasons or epochs.

Our results show:

(1) A strong absorption of UV and visible radiations by Pluto tholins, attributed to their Nbearing and O-bearing organic molecules.

(2) A moderate absorption in the near-IR spectral range by Pluto tholins with $k$ indices of a few $10^{-3}$.

(3) A dependency of $n$ and $k$ indices to the $\mathrm{CH}_{4}: \mathrm{N}_{2}$ mixing ratio, and thus to the altitude of aerosol formation in Pluto's atmosphere. The $\mathrm{N}$ - and O-content in Pluto low-altitude aerosols is enhanced, leading to an increase of both $n$ - and $k$-values. This dependency of the optical constants to the $\mathrm{CH}_{4}: \mathrm{N}_{2}$ mixing ratio has also implications for the seasonal and past epochal radiative transfer of Pluto.

The comparison of our data with the optical constants determined for Titan tholins by Khare et al. (1984) shows that Pluto tholins present globally similar $n$-values to those of Titan. However, 
differences are observed concerning the $k$ indices. In particular, $k$-values of Pluto tholins are one order of magnitude higher than those of Titan in the near-IR. In the UV spectral range, $k$ indices of Pluto tholins are also higher, which is probably due to the reactivity of nitrogen and carbon monoxide in the gas mixture producing Pluto's aerosols.

The optical properties of tholins depend on their chemical composition, and particularly on the $\mathrm{N}$ - and O-content. As Pluto tholins are compositionally different from Titan tholins, we propose the optical constants determined in this study as new input parameters from 270 to $2100 \mathrm{~nm}$ to model Pluto's atmosphere and surface properties.

Our Pluto optical constants were used as input parameters in a Pluto surface model developed by Protopapa et al. (2020). The modelling results concluded on the suitability of our optical constants to reproduce Pluto compositional observations by MVIC and LEISA, at least in the UV-Vis spectral ranges. Moreover, results from a model of light scattering developed by Rannou et al. $(1997,1999)$ concluded that Pluto tholins absorb 5 to 10 times less than Titan tholins at $500 \mathrm{~nm}$. This lower absorption is consistent with Alice observations of Pluto's haze.

Our study focused on Pluto's aerosols formed by photochemistry. However, we remind that an additional process for the formation of aerosols in Pluto's atmosphere was recently proposed by Lavvas et al. (2020) due to ice condensation on photochemical nuclei. In their hypothesis, the first photochemical ice to condense in the upper atmosphere (near $\sim 500 \mathrm{~km}$ of altitude) would be $\mathrm{HCN}$. Then, other photochemical gases, such as $\mathrm{C}_{3} \mathrm{H}_{4}, \mathrm{C}_{6} \mathrm{H}_{6}$ and predominantly $\mathrm{C}_{4} \mathrm{H}_{2}$, would condense and coat the HCN ice particles formerly formed. Note that, as Pluto's most abundant gaseous photochemical products $-\mathrm{C}_{2} \mathrm{H}_{2}, \mathrm{C}_{2} \mathrm{H}_{4}$, and $\mathrm{C}_{2} \mathrm{H}_{6}$ - condense only a few kilometres above the surface (Stern et al. 2017b), they should not take part in the homogeneous coating of $\mathrm{HCN}$ particles occurring at higher altitudes. Nevertheless, those $\mathrm{C}_{2}$ hydrocarbons, as well as $\mathrm{CH}_{3} \mathrm{C}_{2} \mathrm{H}$, are suspected to be subject to heterogeneous adsorption/desorption processes on the ice particles, between 100 and $200 \mathrm{~km}$ of altitude above the surface (Krasnopolsky 2020; Luspay-Kuti et al. 2017; Wong et al. 2017), inhibiting the sublimation of the condensed ices.

Such condensation of gaseous molecules, resulting in coated photochemical aerosols (Krasnopolsky 2020; Luspay-Kuti et al. 2017; Wong et al. 2017), would occur on preexisting Pluto's aerosols formed by photochemistry, requiring to know their properties as well, as studied in the present work. The differences observed between our data and the New Horizons observations could be explained by this contribution of coated aerosols. By analogy with the $\mathrm{C}_{4} \mathrm{H}_{2}$ ice studied in Lavvas et al. (2020) presenting lower $n$ - and $k$-values than those determined 
for our Pluto tholins in the common wavelength range [270-1000 nm], we can suppose that if gaseous molecules condense or adsorb on Pluto's photochemical aerosols, their optical constants $n$ and $k$ could be slightly lowered.

However, assessing the exact effect on the optical constants of [tholins/ices] mixture will require in depth modelling and experimental investigation, far beyond the scope of this study.

\section{Acknowledgements}

Ellipsometric data were acquired at the Ellipsometry core facility of LCP-A2MC, Université de Lorraine - http://lcp-a2mc.univ-lorraine.fr.

L.J. thanks Edith Hadamcik and Guy Cernogora for the insightful proofreading of the manuscript.

N.C., L.J., and T.G. are grateful to the European Research Council Starting Grant PrimChem for funding this work (grant agreement $n^{\circ} 636829$ ).

T.G. acknowledges the support for this work by the Programme National de Planétologie (PNP) of CNRS/INSU, cofunded by CNES.

S.P. thanks the NASA Grant 80NSSC19K0554 for partial funding that supported her work.

T.B. was supported for this research by an appointment to the National Aeronautics and Space Administration (NASA) Post-doctoral Program at the Ames Research Center administered by Universities Space Research Association (USRA) through a contract with NASA.

\section{References}

Alcouffe, G., Cavarroc, M., Cernogora, G., et al. 2010, Plasma Sources Sci Technol, 19, 15008, http://dx.doi.org/10.1088/0963-0252/19/1/015008

Azzam, R. M. A., \& Bashara, N. M. 1977, Ellipsometry and Polarized Light, Ellipsometry and Polarized Light (North-Holland Publishing Company)

Bertrand, T., \& Forget, F. 2016, Nature, 540, 86, https://doi.org/10.1038/nature19337

Bertrand, T., \& Forget, F. 2017, Icarus, 287, 72,

http://www.sciencedirect.com/science/article/pii/S0019103517300477 
863

864

865

866

867

868

869

870

871

872

873

874

875

876

877

878

879

880

881

882

883

884

885

886

887

888

889

890

Bertrand, T., Forget, F., Umurhan, O. M., et al. 2019, Icarus, 329, 148, http://www.sciencedirect.com/science/article/pii/S0019103518305748

Bohren, C. F., \& Huffman, D. R. 1983, Absorption and Scattering of Light by Small Particles (John Wiley \& Sons)

Boucher, O. 2015 (Springer Netherlands), https://doi.org/10.1007/978-94-017-9649-1_2

Brassé, C., Muñoz, O., Coll, P., \& Raulin, F. 2015, Planet Space Sci, 109-110, 159, http://www.sciencedirect.com/science/article/pii/S0032063315000471

Brossier, J. F., Rodriguez, S., Cornet, T., et al. 2018, J Geophys Res Planets, 123, 1089, https://doi.org/10.1029/2017JE005399

Bruggeman, D. A. G. 1935, Ann Phys, 416, 636, https://doi.org/10.1002/andp.19354160705

Cable, M. L., Hörst, S. M., Hodyss, R., et al. 2012, Chem Rev, 112, 1882, https://doi.org/10.1021/cr200221x

Cappa, C. D., Che, D. L., Kessler, S. H., Kroll, J. H., \& Wilson, K. R. 2011, J Geophys Res Atmos, 116, https://doi.org/10.1029/2011JD015918

Carrasco, N., Tigrine, S., Gavilan, L., Nahon, L., \& Gudipati, M. S. 2018, Nat Astron, 2, 489, https://doi.org/10.1038/s41550-018-0439-7

Cechalova, B., Branecky, M., Klapetek, P., \& Cech, V. 2019, Materials, Vol. 12

Cheng, A. F., Summers, M. E., Gladstone, G. R., et al. 2017, Icarus, 290, 112, http://www.sciencedirect.com/science/article/pii/S0019103516303141

Cheng, A. F., Weaver, H. A., Conard, S. J., et al. 2008, Space Sci Rev, 140, 189, https://doi.org/10.1007/s11214-007-9271-6

D’Amico, K. L., Manos, C., \& Christensen, R. L. 1980, J Am Chem Soc, 102, 1777, https://doi.org/10.1021/ja00526a003

Desyaterik, Y., Sun, Y., Shen, X., et al. 2013, J Geophys Res Atmos, 118, 7389, https://doi.org/10.1002/jgrd.50561

Fan, S., Shemansky, D. E., Li, C., et al. 2019, Earth Sp Sci, 6, 1057, https://doi.org/10.1029/2018EA000477

Ferrari, A. C., Rodil, S. E., \& Robertson, J. 2003, Phys Rev B - Condens Matter Mater Phys, 
892 Fujiwara, H. 2007, Spectroscopic Ellipsometry: Principles and Applications, Spectroscopic 893 Ellipsometry: Principles and Applications (John Wiley)

894 Gao, P., Fan, S., Wong, M. L., et al. 2017, Icarus, 287, 116, 895 http://www.sciencedirect.com/science/article/pii/S0019103516306170

896 Gautier, T., Sebree, J. A., Li, X., et al. 2017, Planet Space Sci, 140, 27, 897 http://www.sciencedirect.com/science/article/pii/S003206331630397X

898 Gavilan, L., Broch, L., Carrasco, N., Fleury, B., \& Vettier, L. 2017, Astrophys J, 848, L5, 899 http://dx.doi.org/10.3847/2041-8213/aa8cc4

900 Gladstone, G. R., Pryor, W. R., \& Stern, S. A. 2015, Icarus, 246, 279, 901 http://www.sciencedirect.com/science/article/pii/S0019103514002085

902 Gladstone, G. R., Stern, S. A., Ennico, K. A., et al. 2016, Science (80- ), 351, 903 http://science.sciencemag.org/content/351/6279/aad8866.abstract

904 Griffith, C. A., Doose, L., Tomasko, M. G., Penteado, P. F., \& See, C. 2012, Icarus, 218, 975, 905 http://www.sciencedirect.com/science/article/pii/S0019103511004611

906 Grundy, W. M., Bertrand, T., Binzel, R. P., et al. 2018, Icarus, 314, 232, 907 http://www.sciencedirect.com/science/article/pii/S0019103517306012

908 Hadamcik, E., Renard, J.-B., Alcouffe, G., et al. 2009, Planet Space Sci, 57, 1631, 909 http://www.sciencedirect.com/science/article/pii/S0032063309001834

910 Imanaka, H., Cruikshank, D. P., Khare, B. N., \& McKay, C. P. 2012, Icarus, 218, 247, 911 http://www.sciencedirect.com/science/article/pii/S0019103511004453

912 Imanaka, H., Khare, B. N., Elsila, J. E., et al. 2004, Icarus, 168, 344, 913 http://www.sciencedirect.com/science/article/pii/S0019103504000041

914 Jellison, G. E. J., \& Modine, F. A. 1996, Appl Phys Lett, 69, 371,

915 https://doi.org/10.1063/1.118064

916 Johnson, P. E., Young, L. A., Protopapa, S., et al. 2021, Icarus, 356, 114070,

917 http://www.sciencedirect.com/science/article/pii/S0019103520304188

918 Jovanović, L., Gautier, T., Vuitton, V., et al. 2020, Icarus, 346, 113774, 
https://doi.org/10.1016/j.icarus.2020.113774

Keita, A.-S., En Naciri, A., Delachat, F., et al. 2010, J Appl Phys, 107, 93516, https://doi.org/10.1063/1.3331551

Khare, B. N., Sagan, C., Arakawa, E. T., et al. 1984, Icarus, 60, 127, http://www.sciencedirect.com/science/article/pii/0019103584901428

Khare, B. N., Sagan, C., Thompson, W. R., Arakawa, E. T., \& Votaw, P. 1987, J Geophys Res Sp Phys, 92, 15067, https://doi.org/10.1029/JA092iA13p15067

Kolokolova, L., \& Jockers, K. 1997, Planet Space Sci, 45, 1543, http://www.sciencedirect.com/science/article/pii/S0032063397000998

Kolokolova, L., Jockers, K., Chernova, G. P., \& Kiselev, N. N. 1997, Icarus, 126, 351, http://www.sciencedirect.com/science/article/pii/S0019103596956602

Kramers, H. A. 1927, in Atti. Cong. Intern. Fisica (Transactions of Volta Centenary Congress) Como 2, 545

Krasnopolsky, V. A. 2020, Icarus, 335, 113374, http://www.sciencedirect.com/science/article/pii/S0019103519302313 van Krevelen, D. W., \& te Nijenhuis, K. 2009, in Properties of Polymers - Fourth edition, 287, http://www.sciencedirect.com/science/article/pii/B9780444828774500172

Kronig, R. de L. 1926, J Opt Soc Am, 12, 547, http://www.osapublishing.org/abstract.cfm?URI=josa-12-6-547

Lambe, A. T., Cappa, C. D., Massoli, P., et al. 2013, Environ Sci Technol, 47, 6349, https://doi.org/10.1021/es401043j

Larson, E. J. L., Toon, O. B., West, R. A., \& Friedson, A. J. 2015, Icarus, 254, 122 , http://www.sciencedirect.com/science/article/pii/S0019103515001104

Lavvas, P., Lellouch, E., Strobel, D. F., et al. 2020, Nat Astron, https://doi.org/10.1038/s41550-020-01270-3

Lavvas, P., Strobel, D. F., Lellouch, E., et al. 2016, in AAS/Division for Planetary Sciences Meeting Abstracts \#48, 224.06, https://ui.adsabs.harvard.edu/abs/2016DPS....4822406L

Lavvas, P., Yelle, R. V., \& Griffith, C. A. 2010, Icarus, 210, 832, 
947

948

949

950

951

952

953

954

955

956

957

958

959

960

961

962

963

964

965

966

967

968

969

970

971

972

973

974

http://www.sciencedirect.com/science/article/pii/S0019103510003040

Lavvas, P., Yelle, R. V., \& Vuitton, V. 2009, Icarus, 201, 626, http://www.sciencedirect.com/science/article/pii/S0019103509000086

Lellouch, E., Gurwell, M. A., Butler, B. J., et al. 2017, Icarus, 286, 289, http://www.sciencedirect.com/science/article/pii/S0019103516302755

Liang, M.-C., Yung, Y. L., \& Shemansky, D. E. 2007, Astrophys J, 661, L199, http://dx.doi.org/10.1086/518785

Likhachev, D. V., Malkova, N., \& Poslavsky, L. 2015, Thin Solid Films, 589, 844, http://www.sciencedirect.com/science/article/pii/S0040609015007014

Liu, P. F., Abdelmalki, N., Hung, H.-M., et al. 2015, Atmos Chem Phys, 15, 1435, https://ui.adsabs.harvard.edu/abs/2015ACP....15.1435L

Lopes, R. M. C., Malaska, M. J., Solomonidou, A., et al. 2016, Icarus, 270, 162, http://www.sciencedirect.com/science/article/pii/S0019103515005576

Luspay-Kuti, A., Mandt, K. E., Jessup, K. L., et al. 2017, Mon Not R Astron Soc, 472, 104

Mahjoub, A., Carrasco, N., Dahoo, P.-R., et al. 2012, Icarus, 221, 670, http://www.sciencedirect.com/science/article/pii/S001910351200334X

Mahjoub, A., Carrasco, N., Dahoo, P.-R., et al. 2014, Plasma Process Polym, 11, 409, https://doi.org/10.1002/ppap.201300150

Maillard, J., Carrasco, N., Schmitz-Afonso, I., Gautier, T., \& Afonso, C. 2018, Earth Planet Sci Lett, 495, 185, http://www.sciencedirect.com/science/article/pii/S0012821X18302851

Mistrik, J., Kasap, S., Ruda, H. E., Koughia, C., \& Singh, J. 2017 (Springer International Publishing), https://doi.org/10.1007/978-3-319-48933-9_3

Moise, T., Flores, J. M., \& Rudich, Y. 2015, Chem Rev, 115, 4400, https://doi.org/10.1021/cr5005259

Nakayama, T., Sato, K., Imamura, T., \& Matsumi, Y. 2018, Environ Sci Technol, 52, 2566, https://doi.org/10.1021/acs.est.7b05852

Nakayama, T., Sato, K., Matsumi, Y., et al. 2012, SOLA, 8, 119

Pernot, P., Carrasco, N., Thissen, R., \& Schmitz-Afonso, I. 2010, Anal Chem, 82, 1371, 
975

976

977

978

979

980

981

982

983

984

985

986

987

988

989

990

991

992

993

994

995

996

997

998

999

1000

1001

1002

https://doi.org/10.1021/ac902458q

Protopapa, S., Grundy, W. M., Reuter, D. C., et al. 2017, Icarus, 287, 218, http://www.sciencedirect.com/science/article/pii/S0019103516307692

Protopapa, S., Olkin, C. B., Grundy, W. M., et al. 2020, Astron J, 159, 74, http://dx.doi.org/10.3847/1538-3881/ab5e82

Quirico, E., Montagnac, G., Lees, V., et al. 2008, Icarus, 198, 218, http://www.sciencedirect.com/science/article/pii/S0019103508002819

Ramírez, S. I., Coll, P., da Silva, A., et al. 2002, Icarus, 156, 515, http://www.sciencedirect.com/science/article/pii/S0019103501967831

Rannou, P., Cabane, M., Botet, R., \& Chassefière, E. 1997, J Geophys Res Planets, 102, 10997, https://doi.org/10.1029/97JE00719

Rannou, P., Cours, T., Le Mouélic, S., et al. 2010, Icarus, 208, 850,

http://www.sciencedirect.com/science/article/pii/S0019103510001181

Rannou, P., McKay, C. P., Botet, R. S., \& Cabane, M. 1999, Planet Space Sci, 47, 385, http://www.sciencedirect.com/science/article/pii/S0032063399000070

Rao, C. N. R. 1975 (Butterworth)

Reuter, D. C., Stern, S. A., Scherrer, J., et al. 2008, Space Sci Rev, 140, 129, https://doi.org/10.1007/s11214-008-9375-7

Scattergood, T., \& Owen, T. 1977, Icarus, 30, 780, http://www.sciencedirect.com/science/article/pii/0019103577900951

Sciamma-O’Brien, E., Dahoo, P.-R., Hadamcik, E., et al. 2012, Icarus, 218, 356, http://www.sciencedirect.com/science/article/pii/S0019103511004878

Seignovert, B., Rannou, P., Lavvas, P., Cours, T., \& West, R. A. 2017, Icarus, 292, 13, http://www.sciencedirect.com/science/article/pii/S0019103516306911

Steffl, A. J., Young, L. A., Strobel, D. F., et al. 2020, Astrophys J

Stenberg, B., Viscarra Rossel, R. A., Mouazen, A. M., \& Wetterlind, J. 2010, in Advances in Agronomy, ed. D. L. B. T.-A. in A. Sparks, Vol. 107 (Academic Press), 163, http://www.sciencedirect.com/science/article/pii/S0065211310070057 
Stern, S. A., Bagenal, F., Ennico, K. A., et al. 2015, Science (80- ), 350, http://science.sciencemag.org/content/350/6258/aad1815.abstract

Stern, S. A., Binzel, R. P., Earle, A. M., et al. 2017a, Icarus, 287, 47, http://www.sciencedirect.com/science/article/pii/S0019103516307588

Stern, S. A., Grundy, W. M., McKinnon, W. B., Weaver, H. A., \& Young, L. A. 2018, Annu Rev Astron Astrophys, 56, 357, https://doi.org/10.1146/annurev-astro-081817-051935

Stern, S. A., Kammer, J. A., Barth, E. L., et al. 2017b, Astron J, 154, 43, http://dx.doi.org/10.3847/1538-3881/aa78ec

Stern, S. A., Slater, D. C., Scherrer, J., et al. 2008, Space Sci Rev, 140, 155, https://doi.org/10.1007/s11214-008-9407-3

Szopa, C., Cernogora, G., Boufendi, L., Correia, J.-J., \& Coll, P. 2006, Planet Space Sci, 54, 394, http://www.sciencedirect.com/science/article/pii/S003206330600002X

Tauc, J., Grigorovici, R., \& Vancu, A. 1966, Phys Status Solidi, 15, 627, https://doi.org/10.1002/pssb.19660150224

Tompkins, H. G., \& Irene, E. A. 2005, Handbook of Ellipsometry (William Andrew Publishing)

Tran, B. N., Joseph, J. C., Ferris, J. P., Persans, P. D., \& Chera, J. J. 2003, Icarus, 165, 379, http://www.sciencedirect.com/science/article/pii/S0019103503002094

Vinatier, S., Rannou, P., Anderson, C. M., et al. 2012, Icarus, 219, 5, http://www.sciencedirect.com/science/article/pii/S0019103512000498

Vuitton, V., Tran, B. N., Persans, P. D., \& Ferris, J. P. 2009, Icarus, 203, 663, http://www.sciencedirect.com/science/article/pii/S0019103509001754

Wong, M. L., Fan, S., Gao, P., et al. 2017, Icarus, 287, 110, http://www.sciencedirect.com/science/article/pii/S0019103516306145

Workman, J., \& Weyer, L. 2008, Practical Guide to Interpretive Near-Infrared Spectroscopy, Vol. 3

Xiaobo, Z., Jiewen, Z., Povey, M. J. W., Holmes, M., \& Hanpin, M. 2010, Anal Chim Acta, 667, 14, http://www.sciencedirect.com/science/article/pii/S0003267010003582 
Young, L. A., Kammer, J. A., Steffl, A. J., et al. 2018, Icarus, 300, 174, http://www.sciencedirect.com/science/article/pii/S0019103517302609

Zhang, X., Strobel, D. F., \& Imanaka, H. 2017, Nature, 551, 352,

https://doi.org/10.1038/nature24465

Zhong, M., Jang, M., Oliferenko, A., Pillai, G. G., \& Katritzky, A. R. 2012, Phys Chem Chem Phys, 14, 9058, http://dx.doi.org/10.1039/C2CP23906J

Zhu, X., Strobel, D. F., \& Erwin, J. T. 2014, Icarus, 228, 301, http://www.sciencedirect.com/science/article/pii/S0019103513004302

\section{Supplementary Information}

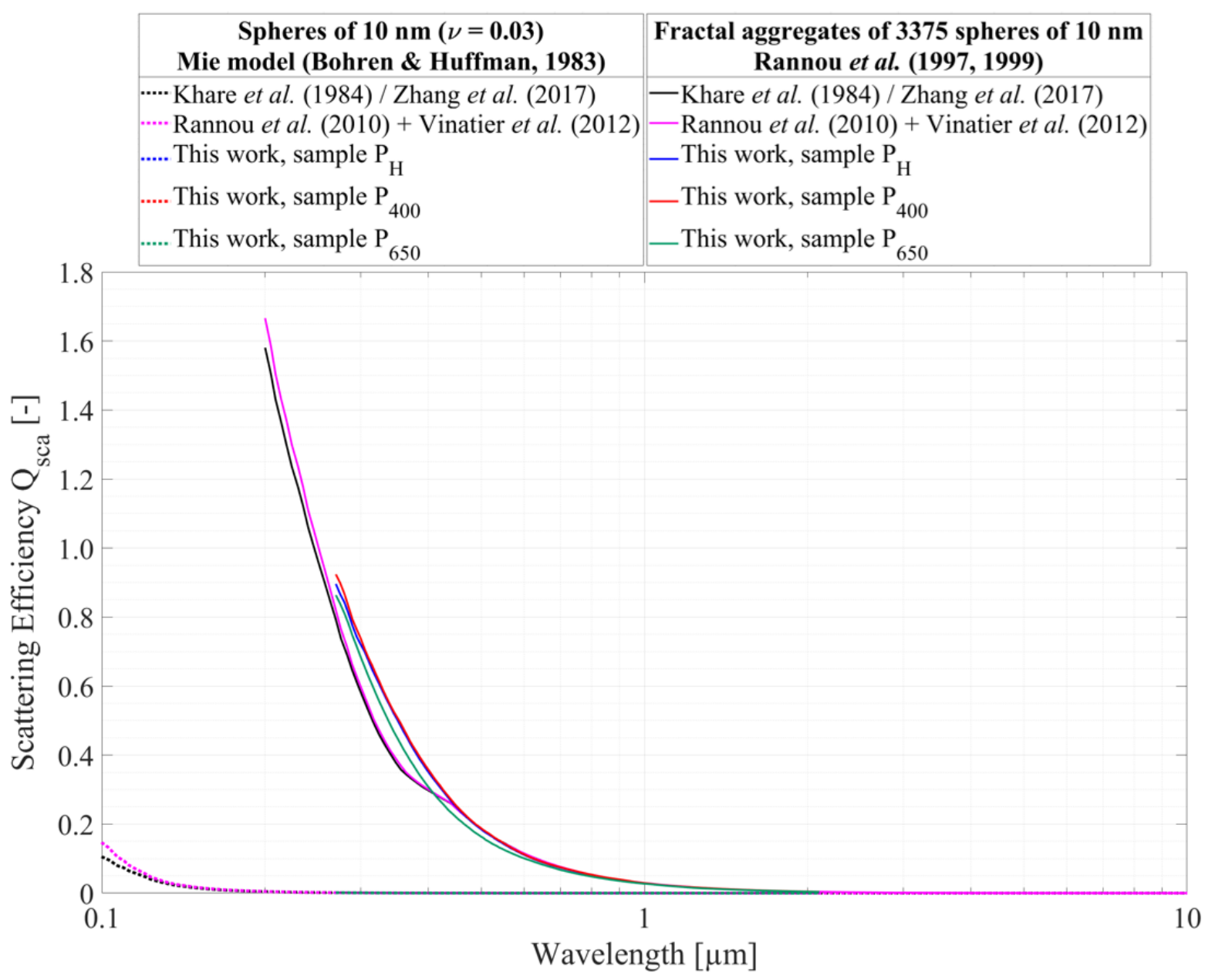

Figure A: Scattering efficiency $\mathrm{Q}_{\text {sca }}$ determined for spherical monomers of $10 \mathrm{~nm}$ radius (lognormal distribution, with effective variance $v=0.03$ ) with a Mie model formalized in Bohren 
\& Huffman (1983) (dotted curves) and for fractal aggregates of 3375 monomers of $10 \mathrm{~nm}$ radius with the model developed by Rannou et al. $(1997,1999)$ (plain curves). The black curves were generated with the optical constants determined by Khare et al. (1984) for Titan tholins. Note that these Khare et al. (1984) optical constants were adopted in Zhang et al. (2017) study. The pink curves were generated with the optical constants determined by Rannou et al. (2010) and Vinatier et al. (2012) for Titan's aerosols. The blue, red and green curves were generated with the optical constants determined respectively for our samples $\mathrm{P}_{\mathrm{H}}, \mathrm{P}_{400}$ and $\mathrm{P}_{650}$.

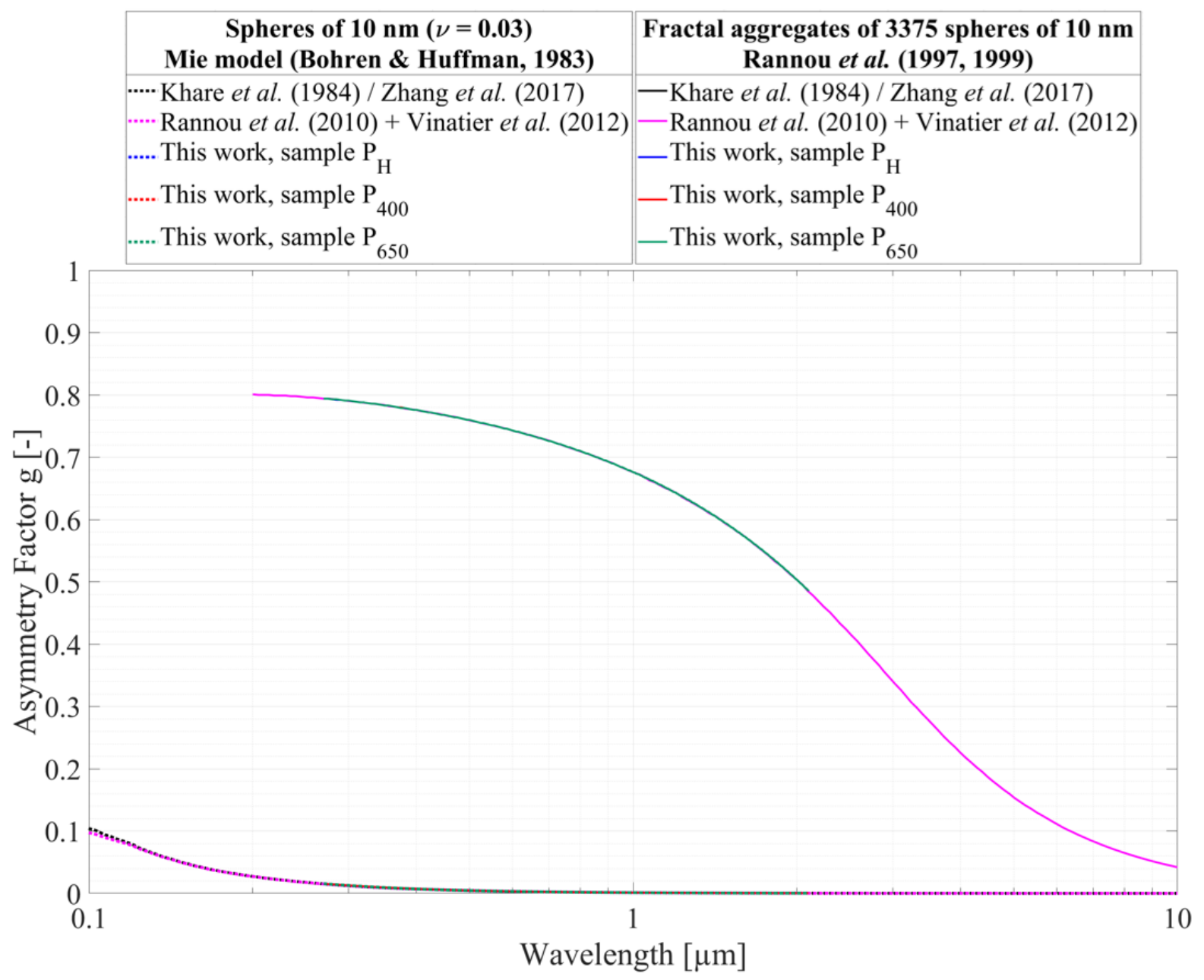

Figure B: Asymmetry factor g determined for spherical monomers of $10 \mathrm{~nm}$ radius (log-normal distribution, with effective variance $v=0.03$ ) with a Mie model formalized in Bohren \& Huffman (1983) (dotted curves) and for fractal aggregates of 3375 monomers of $10 \mathrm{~nm}$ radius with the model developed by Rannou et al. $(1997,1999)$ (plain curves). The black curves were generated with the optical constants determined by Khare et al. (1984) for Titan tholins. Note that these Khare et al. (1984) optical constants were adopted in Zhang et al. (2017) study. The pink curves were generated with the optical constants determined by Rannou et al. (2010) and 
1060 Vinatier et al. (2012) for Titan's aerosols. The blue, red and green curves were generated with 1061 the optical constants determined respectively for our samples $\mathrm{P}_{\mathrm{H}}, \mathrm{P}_{400}$ and $\mathrm{P}_{650}$. 